\title{
Répartition de l'énergie dans des zones de décollement
}

\author{
par Hunter ROUSE \\ IOWA INSTITUTE OF HYDRAULIG RESEARCH \\ STATE UNIVERSITY OF IOWA
}

Au cours des dix dernieres années, l'Institut de Recherches hydrauliques d'lowa a entrepris une série d'études de l'écoulement au voisinage des tourbillons quasi stables produits par le décollement au voisinage de parois anguleuses.

Le professeur Rouse expose dans le présent mémoire le mode de détermination des écoulements axisymétriques moyen et secondaire pour deux parois de formes comparables: une entrée brusque de conduite et un cylindre tronqué.

Les mesures ulilisables pour l'analyse comprennent les répartitions de la vitesse molyenne et de la pression moljenne, les intensités axiale et radiale de la turbulence, les contraintes de cisaillement turbulent et le gradient d'intensité axiale. Au moyen des équations de la quantité de mouvement ef de l'énergie pour les mouvements moyen el secondaire, les répartitions mesurées sont rectifiés de façon ì obtenir l'égalité nécessaire des termes essentiels des équations, fournissant ainsi des résultats concordant généralement avec les conditions physiques. Ceux-ci sont eux-mêmes présentés sous forme de schémas de l'écoulement, accompagnés de courbes donnant la variation de l'énergie et des quantités de. mouvement individuelles dans loutes les zones de décollement.

Une importance particulière s'attache à la relation entre Une importance particulière s'attache à la relation entre
la production de la turbulence, la convection et la dissipation et le fait qu'wne quantité relativement faible de l'énergie de la turbulence est localement nécessaire pour amener un changement total dans ia configuration de l'écoulement.

Enfin, deux diagranmes montrent la variation des termes de l'équation de Bernonlli étendue jusqu'à inclure les effets de la turbulence et de la dissipation de l'énergie.

English text, p. 235

\section{INTRODUCTION}

Dans le domaine de l'hydraulique, le décollement local de l'écoulement principal d'avec la paroi est une caractéristique très connue des phénomènes. Soit pour raison d'économie, par indifférence du projeteur, ou pour cause d'impraticabilité technique, l'usage de profils aérodynamiques, pour éviter ce phénomène, est toujours l'exception plutôt que la règle. Des éléments de plomberie, des raccordements de canaux, des entrées de conduites et des piles de ponts ont ainsi souvent un profil coudé plutôt cu'arrondi, où le décollement est habituellement la cause de vibrations, de pertes de charge, ou d'érosion. Mais il est vrai que, parfois, le décollement et la formation de tourbillons sont des aspects inévitables du mode d'écoulement, comme dans le cas des vannes, des chicanes et des bassins d'amortissement. En outre, les profils de parois coudés sont en certains cas préférables à ceux insuffisamment arrondis, parce que ceux-ci peuvent provoquer des intermittences dans le processus de décollement, lequel n'a presque rien en sa faveur et beaucoup contre lui. En tout cas, la mécanique de l'écoulement dans une zone de décollement semblerait en beaucoup de cas être plus importante pour l'hydraulicien que pour tout autre savant ou ingénieur. C'est peut-être pourquoi de tels écoulements ont trouvé jusqu'à maintenant aussi peu d'attention sur le plan théorique. Cependant, il y a là une bonne raison pour l'hydraulicien de prendre une part importante dans son étude.

L'hydraulique s'est depuis longtemps intéressée presque exclusivement aux analyses de l'écoulement moyen, et en conséquence, elle a négligé le mouvement secondaire ou turbulent qui se superpose au mouvement primaire ou moyen. Dans les dernières années, d'autres professions ont apporté beaucoup d'attention à la turbulence même, en limitant le phénomène à tel point que l'écoulement moyen pouvait être négligé. Au moyen de telles simplifications, on a beaucoup appris sur les écoulements moyens et sur les bases de la turbulence. Dans la plupart des états du mouvement fluide réel, cependant, ni l'un ni l'autre de ces aspects ne peut être étudié tout seul d'une manière satisfaisante, car chacun a un rôle déterminant des caractéristiques de l'autre. Et cela est spécialement vrai pour le phénomène de décollement vu sous ses aspects les plus étendus.

Des progrès considérables ont été faits dans l'aérodynamique en prévoyant le point de décollement sur des parois progressivement courbées en fonction de la géométrie des parois et $\mathrm{du}$ nombre de Reynolds - pourvu que le décollement n'ait aucun effet appréciable sur l'écoulement voisin, ou l'affecte simplement d'une manière qui peut être évaluẻe par l'analyse des lignes de jet. Cependant, en cas de parois simples, comme celles que montre la figure 1 , dans

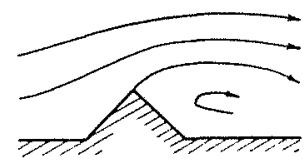

F1G. 1

Décollement à partir d'une irrégularité de paroi Separation at a boundary irregularity 
laquelle le point de décollement est fixé, non seulement il est impossible de prévoir avec exactitude la forme moyenne de la zone de décollement, mais encore, même avec cette connaissance, on ne pourrait estimer qu'approximativement la répartition de la vitesse et de la pression dans le tourbillon et la perte d'énergie qui en résulte; et d'autres caractéristiques de l'écoulement secondaire, telles que l'intensité de la turbulence dans et derrière le tourbillon et la fréquence des variations à son extrémité postérieure, pourraient seulement faire l'objet d'hypothèses.

Le rôle de tels tourbillons dans la transformation de l'énergic a été depuis longtemps sousestimé par les uns, et surestimé par les autres. Nécessairement, le schéma des lignes de courant de l'écoulement moyen montre un vortex stable, avec un système de lignes aussi bien défini que celui du courant extérieur, et on est tenté d'attribuer au tourbillon la même quantité d'énergie par unité de volume que celle de l'écoulement propre. C'est une grave erreur, car la ligne de décollement n'est pas une ligne de courant libre, mais une ligne au long de laquelle l'énergie décroît sans cesse. Cependant, puisque le tourbillon est en effet une source variable de tourbillons plus petits perdus dans son sillage, il est fréquemment représenté comme une sorte de « moulin à tourbillons» dans lequel il se produit de la turbulence et par suite, une perte d'énergie. En réalité, la zone primaire de production de la turbulence se trouve entre le tourbillon et l'écoulement même, comme dans tous les cas de mélange à travers une surface de vitesse discontinue, et la turbulence est convoyée et diffusée avant que son énergie soit tout à fait dissipée. C'est l'écoulement inversé qui distingue ce phénomène des cas plus simples de mélange: d'une part, le fluide entraîné du côté du tourbillon est déjà fortement turbulent; d'autre part, l'extrémité aval du tourbillon change de position pendant que d'appréciables quantités se déchargent de façon intermittente dans l'écoulement propre.

Le problème est évidemment bien loin des deux états du mouvement avec lequel on a déjà obtenu des succès analytiques. Il diffère du cas isotrope, représenté par des sections normales de l'écoulement en aval d'une grille, parce qu'il constitue un problème d'écoulement fortement anisotrope avec des tensions tangentielles; et il diffère du cas de similitude, symbolisé par l'écoulement établi dans un sillage ou une conduite, parce qu'il est spécifiquement un phénomène localisé, bien que son effet s'étende loin en aval. Faute d'instruments analytiques sûrs, l'élude du phénomène doit procéder d'une manière quasi- empirique : il faut examiner des cas caractéristiques avec autant de détails expérimentaux que possible; apprécier les valeurs des quantitités non encore mesurables en comparant les mesures possibles et les équations du mouvement; et essayer, lorsque le mécanisme du tourbillon devient mieux connu, d'en donner l'expression sous une forme mathématique approchée.

Sous notre direction, plusieurs études d'exploration de ce genre ont déjà été faites dans les Laboratoires de l'Institut des Recherches Hydrauliques de Iowa. La première fut un projef de doctorat, dressé par H.C. Hsu [1] sur le décollement en aval d'un élargissement brusque dans une conduite à deux dimensions; les caractéristiques de l'écoulement moyen et de la turbulence furent mesurées et rattachées aux équations du mouvement, de telle sorte que le passage de l'énergie de l'écoulement primaire à l'écoulement secondaire et jusqu'à sa dissipation thermique, put être représentée en gros. Celle-ci fut suivie par une étude de M. Arie [2] sur l'écoulement derrière une paroi perpendiculaire [3], dont les résultats furent analysés sous forme énergétique [4]. Des études semblables, quoique moins dépendantes de la géométrie des parois, furent celles conduites par O. Erickson et nous-même sur un jet normal à un écoulement transversal [5], par T.T. Siao [6] et S. Nagaratnam [7] sur le ressaut hydraulique [8], et par T.K. Rao [9] sur un jet dirigé axialement contre un écoulement. Chacun de ces cas permet de constater l'existence commune d'une zone stable d'écoulement inversé — un tourbillon stationnaire — dont la turbulence était engendrée le long des bords; la convection, la diffusion et la dissipation consécutives à cette turbulence furent des facteurs essentiels de détermination de la forme de l'écoulement entier.

La thèse suivante s'avance davantage vers la compréhension finale de ce phénomène; elle décrit une étude de l'écoulement en symétrie axiale auprès de deux configurations comparables de parois, d'intérêt fondamental en hydraulique et dans les domaines voisins : une entrée de conduite et un cylindre à bout trempé. Les mouvements primaire et secondaire sont étudiés, en s'efforçant de déterminer le processus du transfert d'énergie depuis l'écoulement moyen, par la turbulence jusqu'à sa forme finale, la chaleur. En prenant ces écoulements comme exemples, la signification des relations entre les formes d'ènergie différentes est discutée en détail dans le but de faciliter à l'avenir de semblables études. 


\section{EXAMEN PRELIMINAIRE DES ECOULEMENTS A ETUDIER}

Une entrée brusque dans une conduite située dans la paroi d'un grand réservoir et un cylindre de section normale sont des parois qui semblent au premier coup d'weil bien différentes l'une de l'autre, car l'une représente les voies par lesquelles un fluide s'écoule et l'autre les corps qui sont transportés généralement par les fluides. Bien entendu, le principe du mouvement relatif élimine facilement l'une des deux différences apparentes. Pourtant, quant à l'autre, la véritable similitude entre les deux espèces de parois ne devient apparente qu'après les avoir étudices en fonction de leur's homologues à deux dimensions. En fait, une telle représentation a un double but, puisque seules les images planes sont, même approximativement, susceptibles d'être soumises à l'analyse mathématique, et cette approximation est souhaitable au moins pour comprendre qualitativement les principes de physique qui sont à la base des écoulements respectifs.

En examinant la figure 2, on voit que les élé-

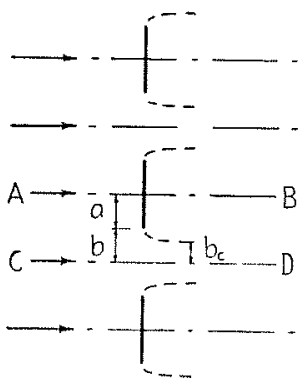

FIG. 2

Ecoulement par une série de plaques normales

Flow past a series of normal plates

ments multiples de l'écoulement autour d'une série de plaques à arêtes vives peuvent être considérés de deux manières différentes. Ainsi l'élément ABCD se combine au-dessus avec son image pour former un passage symétrique en relation avec la ligne $A B$ représentant alors l'écoulement renfermé autour d'une plaque; la ligne $\mathrm{CD}$ et son image marquent la position des parois solides. De même, on peut considérer CD comme axe de symétrie, avec des parois solides le long de $A B$ et de son image, ainsi que les deux demi-plaques, tout en simulant un passage avec un étranglement local de chaque côté. $\mathrm{Si}$, pour un instant, on néglige le mélange des sillages voisins en aval, les lignes de décollement sembleraient être les mêmes dans chaque cas. La verritable distinction entre les deux points de vue ne devient évidente que lorsque la demi-épaisseur $a$ et la demi-distance $b$ entre les plats deviennent de plus en plus dif-férentes. Lorsque le rapport $a / b$ devient très inférieur à l'unité, par exemple, c'est l'aspect de corps immergé de cet arrangement qui est accentué; et quand le rapport devient très grand, c'est l'aspect de l'écoulement qui prédomine. N'aturellement, la limite $a / b=0$ du premier cas est la plaque de dimension finie dans un fluide infini, alors que la limite $a / b=\infty$ de l'autre cas est une fente de dimension finie dans une paroi infiniment grande.

En utilisant la méthode de l'analyse conforme, avec l'hypothèse de lignes de courant libres, von Mises [10] put évaluer le rapport $b_{c} / b$ de contraction du jet pour toutes les valeurs du rapport $a / b$ des parois, y compris, bien entendu, la limite de Kirchhoff :

$$
\pi /(\pi+2)=0,61
$$

pour la valeur $a / b=\infty$. Ces résultats ont été bien approchés par des expériences avec des jets libres aussi bien qu'immergés. Cependant, l'application de la même méthode d'analyse à l'écoulement autour d'une plaque donne des résultats qui ne sont vérifiés que pour une interface entre un liquide et un gaz (c'est-à-dire une poche de cavitation) de grande étenduc, car c'est seulement en ce cas que la pression dans la poche est proche de celle de l'écoulement ambiant. Cependant, conme l'a démontré Riabouchinsky [11], l'hypothèse soit d'une pression plus basse soit d'une courbure plus rapide permettra à des méthodes d'analyse de donner des résultats plus conformes aux mesures.

Bien sûr, une telle concordance est seulement approchée, car elle dépend des forces ou des variations de pression plus que des particularités locales de l'écoulement. Celles-ci, en effet, sont tout à fait contraires à l'hypothèse de la ligne de courant libre, sauf au voisinage immédiat du point de décollement. Par suite des tensions tangentielles élevées le long de la surface libre du décollement, sauf aux faibles nombres de Reynolds, e'est ici le siège d'une intense production de turbulence, laquelle diffuse transversalement pendant qu'elle se transporte en aval avec l'écoulement et en même temps modifie les particularités d'une zone devenant toujours plus large. Dans les cas limites $a_{f} / b=0$ et $a / b=\infty$, les conditions sont celles, respectivement, du sillage croissant et du jet croissant, dont les solutions approchées sont connues depuis plusieurs 
décennies [12]. La base de telles solutions est la similitude, observée expérimentalement, de la répartition de la vitesse en sections successives bien en aval des irrégularités initiales. Bien entendu, dans leur voisinage immédiat, les conditions de similitude n'existent ni dans un cas ni dans l'autre.

Des considérations semblables s'appliquent à l'écoulement à travers la cascade de barres, montrée dans la figure 3 , car, dans l'une des

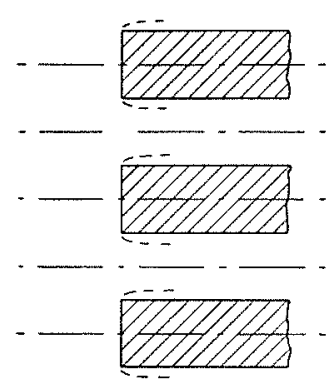

Fra. 3

Ecoulement par une série de barres parallèles Flow past a series of parallel bars

limites, on peut supposer qu'elle représente l'écoulement d'un réservoir dans une conduite uniforme, et à l'autre limite, l'écoulement non confiné autour d'un mur ou d'une pile isolés. Finalement, elles aussi mènent à des états de mouvement qui ont été analysés avec succès en fonction des répartitions de similitude dont l'écoulement plat et uniforme est l'une des limites, et l'autre, le développement d'une couche limite le long d'une surface plane. Au voisinage immédiat des sections initiales, cependant, la similitude n'existe pas, et les méthodes conformes n'ont pas d'autre utilité qu'un sens qualitatif. Par contre, il est évident que les deux états de mouvement ont des traits communs, car, dans chacun, il faut que la poche de décol-

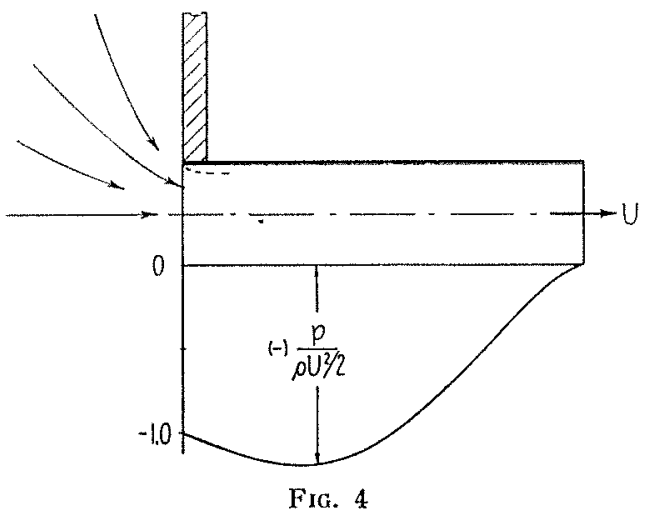

Répartition de la pression pour l'écoulement hors d'un tube court Distribution of pressure for effux through a short tube

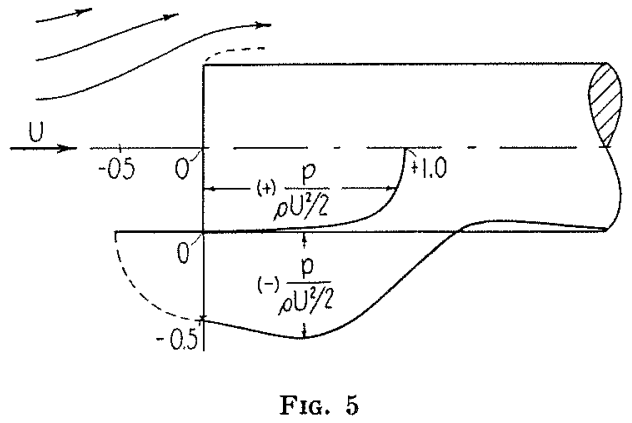

Répartition de la pression

pour l'écoulement autour d'un cylindre tronqué Distribution of pressure for flow around a blunt shaft

lement ait une longueur finie, et que les conditions entre la paroi et le bord de la poche soient essentiellement pareilles. En effet, c'est en dehors de la zone de décollement que la différence fondamentale se trouve, car au-delà de l'une il y a une grande étendue de fluide relativement non perturbé, tandis qu'en face de l'autre existe la même zone de décollement en image réfléchie.

Si l'on examine ensuite les homologues de ces schémas en symétrie axiale (fig. 4 et 5), on verra que les similarités pertinentes dans les cas bidimensionnels sont toutes retenues, tandis que les dissimilarités deviennent encore plus accentuées. Evidemment, les cas de symétrie axiale ne peuvent plus être considérés comme les limites d'un cas paramétrique; au contraire, chacun est en effet la version inverse de l'autre. Bien que ces cas soient encore moins sujets à l'analyse rigoureuse que leurs homologues en deux dimensions, les mêmes observations générales leur sont applicables, et on verra que d'autres encore pourront être trouvées.

Si l'on néglige l'effet de la viscosité le long de la paroi et la déviation croissante des lignes de courant d'un cours purement linéaire, un débit $Q$ à travers une ouverture circulaire dans la paroi d'un réservoir peut ètre défini au moyen de la fonction de courant d'une source négative,

$$
\psi=\frac{Q}{2 \pi} \frac{x}{\sqrt{x^{2}+r^{2}}}
$$

pour laquelle les composantes de la vitesse sont, comme d'habitude, $u=\partial \psi / r \partial r$ dans la direction axiale $x$, et $v=-\partial \psi / r \partial x$ dans la direction radiale $r$. Puisque l'écoulement est rapidement accéléré, l'effet de la viscosité peut être, en fait, négligé sans erreur appréciable, mais la déviation des lignes de courant devient assez importante dans le voisinage de la zone d'émission. Ce désaccord pourrait être éliminé en partie au moyen de la fonction de courant pour une répartition de sources négatives sur le plan de l'ouverture; pourtant, la connaissance expérimentale 
du schéma de l'écoulement (ou au moins de la surface du décollement) est nécessaire pour déterminer la répartition cherchée, et le sujet est par conséquent d'un intérêt plutôt académique que pratique. En revanche, il est connu, grâce à des études numériques [13], que les coefficients de contraction pour des orifices circulaires, considérés comme rapports d'aire, sont à peu près, sinon même exactement, égaux à ceux des fentes ayant les mêmes proportions linéaires; à la limite, par exemple, $\left(d_{t} / d\right)^{2}=0,61$. De plus, l'expérience [14] a montré que cette valeur est presque aussi exacte pour le jet immergé que pour le jet libre. En supposant que le même degré de contraction soit accusé par l'écoulement à l'entrée d'une conduite, la perte de charge à Ja Borda résultante :

$$
\Delta H=\frac{\left(U_{1}-U_{2}\right)^{2}}{2 g}
$$

a été, pendant presque un siècle, combinée avec le coefficient de contraction limite :

$$
\left(\frac{d_{c}}{d}\right)^{2}=0,61=\frac{U}{U_{c}} \approx \frac{U_{2}}{U_{1}}
$$

pour donner comme perte de charge à l'entrée la valeur :

$$
\frac{\Delta H}{U^{2} / 2 g}=1-0,61 \approx 0,5
$$

qui est utilisée d'habitude dans les projets d'hydraulique. Sauf pour plusieurs mesures de la répartition de pression dans un tuyau court, reproduites dans la figure 4 [15], et d'innombrables mesures de pression et de vitesse dans les conduites, loin de l'entrée [16], il semble qu'on en connaît un peu plus sur l'écoulement dont il s'agit.

On connaît encore moins l'écoulement axial autour d'un cylindre à section droite, au moins du point de vue historique, car c'est seulement à propos de la cavitation des projectiles qu'ont été faites des recherches, et cela seulement dans les dernières décennies. Si, pourtant, on conduit la discussion de la même manière, on peut remarquer [17] ceci : les mesures ont montré que la zone de décollement en aval d'un disque a un diamètre environ 1,7 fois celui du disque, le coefficient de traînée a une valeur :

$$
\frac{F /\left(\pi d^{2} / 4\right)}{\rho U^{2} / 2}=1,20
$$

et le coefficient de pression du sillage immédiat est :

$$
\frac{p}{\rho U^{2} / 2}=-0,45
$$

Les mesures de la répartition de pression le long du cylindre [18] sont comme il est indiqué dans la figure 5 où le diamètre de la poche est $1,5 d$, et le coefficient de pression minimum - 0,62. Si la tension tangentielle à la surface du cylindre est négligée, le coefficient de traînée du cylindre doit être au plus 0,78 résultant seulement de la pression sur la face en amont. Pour les sections du sillage sur lesquelles la décroissance de la pression est négligeable, le coefficient de traînée doit correspondre à l'intégrale de la quantité de mouvement [19]:

$$
F=2 \pi \rho \int_{a / 2}^{\infty} u(U-u) r d r
$$

Si la forme de la zone de décollement et de la répartition de vitesse subséquente était connue mème approximativement, de telle sorte que l'épaisseur du déplacement de la perturbation puisse être calculée, la configuration de l'écoulement irrotationnel environnant (sauf peut-être au voisinage immédiat de la face tronquée) pourrait être exprimée par la fonction de courant pour une répartition de doublets $[3,19]$ de puissance $\Delta(t)$ par unité de longueur le long de l'axe de symétrie depuis $x=t=a$ jusqu'à $b$ :

$$
\psi=r^{2} U\left\{1 / 2-\int_{a}^{b} \frac{\Delta(t)}{\left[(t-x)^{2}+r^{2}\right]^{3 / 2}} d t\right\}
$$

Evidemment, cela exigerait, de nouveau, beaucoup de données expérimentales, quoique la partie limite soit connue pour la couche limite le Iong d'une paroi cylindrique [20].

Les deux cas d'écoulement dont il s'agit sont évidemment assujettis à l'analyse mathématique dans les régions irrotationnelles en amont, à la condition que certaines valeurs déterminantes soient fournies par des expériences; de plus, une connaissance considérable - analytique autant qu'empirique - existe déjà pour les zones rotationnelles de similitude bien en aval. Jusqu'a ce jour, néanmoins, on n'a pu obtenir qu'ụne idée qualitative de la transition entre les zones initiale et finale de l'écoulement, sans la mesurer en détails. Les phénomènes de production et de dissipation de la turbulence sont bien connus pour être extrêmement complexes et sauf si l'on emploie à tout le moins les équations hydrodynamiques de base, une telle mesure serait certainement un procédé hasardeux. G'est pourquoi, beaucoup d'attention sera apportée dans la suite à la validité de ces équations. 


\section{FORMULATION ET INTERPRETATION DES EQUATIONS D'ENERGIE}

\section{Equations de Stokes et de Reynolds}

Les équations fondamentales du mouvement d'un fluide en coordonnées cylindriques $x, r$ et 0 se dérivent en égalant à la variation totale des composantes de la vitesse $u, v$ et $w$, les composantes respectives de la force par unité de masse agissant sur un élément fluide tel que celui montré

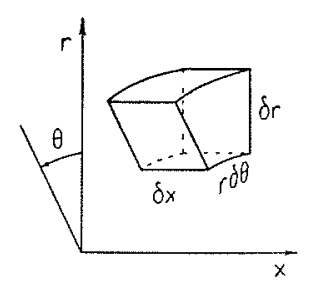

FIG. 6

Notation des coordonnées cylindriques

Cylindrical coordinate notation

par la figure 6. Lorsque le volume de l'élément approche de la limite zéro, ces équations se réduisent à la forme [21]:

$$
\left.\begin{array}{l}
\frac{\partial u}{\partial t}+u \frac{\partial u}{\partial x}+v \frac{\partial u}{\partial r}+w \frac{\partial u}{r \partial \theta}=-\frac{\partial \Omega}{\partial x}+\frac{1}{r}\left[\frac{\partial\left(r \sigma_{x}\right)}{\partial x}+\frac{\partial\left(r \tau_{r x}\right)}{\partial r}+\frac{\partial\left(r \tau_{\theta x}\right)}{r \partial \theta}\right] \\
\frac{\partial v}{\partial t}+u \frac{\partial v}{\partial x}+v \frac{\partial v}{\partial r}+w \frac{\partial v}{r \partial \theta}-\frac{w^{2}}{r}=-\frac{\partial \Omega}{\partial r}+\frac{1}{r}\left[\frac{\partial\left(r \tau_{x r}\right)}{\partial x}+\frac{\partial\left(r \sigma_{r}\right)}{\partial r}+\frac{\partial\left(r \tau_{\theta r}\right)}{r \partial \theta}-\sigma_{\theta}\right] \\
\frac{\partial w}{\partial t}+u \frac{\partial w}{\partial x}+v \frac{\partial w}{\partial r}+w \frac{\partial w}{r \partial \theta}+\frac{v w}{r}=-\frac{\partial \Omega}{r \partial \theta}+\frac{1}{r}\left[\frac{\partial\left(r \tau_{x \theta}\right)}{\partial x}+\frac{\partial\left(r \tau_{r \theta}\right)}{\partial r}+\frac{\partial\left(r \sigma_{\theta}\right)}{r \partial \theta}+\tau_{r \theta}\right]
\end{array}\right\}
$$

Ici $\Omega$ représente la fonction «potentiel dû à la pesanteur» (hauteur multipliée par le poids effectif du fluide par unité de masse); $\sigma_{x}$ est la tension normale par unité de superficie sur une surface perpendiculaire ’̀ l'axe des $x$, nécessairement dans la direction $x$; $\tau_{r: x}$ est la tension tangentielle par unité de superficie sur une surface perpendiculaire à l'axe des $r$ dans la direction $x$, etc. Pour des raisons d'équilibre, $\tau_{r x}=\tau_{x r}$, etc. Aux équations de mouvement doit êtré ajoutée celle de continuité,

$$
\frac{\partial(r u)}{\partial x}+\frac{\partial(r v)}{\partial r}+\frac{\partial(r w)}{r \partial \theta}=0
$$

qui se dérive en exprimant ou bien le fait que le volume d'un élément fluide de densité $\rho$ constante ne doit pas changer pendant qu'il se déplace d'un point à l'autre, ou bien, ce qui équivaut, que l'écoulement instantané qui sort d'une zone élémentaire de l'espace est simultanément égal à l'écoulement qui y entre.

Il y a à peu près un siècle que Stokes [22] exprima les tensions précédentes en fonction de la viscosité dynamique $\mu$, comme suit :

$$
\begin{aligned}
& \sigma_{n}=-p+2 \mu \lambda \quad \sigma_{r}=-p+2 \mu . b \quad \sigma_{\theta}=-p+2 \mu c \\
& \tau_{r, \theta}=\mu, f \quad \tau_{\theta, x}=\mu, g \quad \tau_{r r}=\mu h
\end{aligned}
$$

Ici, $p$ représente l'intensité moyenne de la pression :

$$
p=-\frac{\sigma_{r}+\sigma_{r}+\sigma_{\theta}}{3}
$$

et les lettres $a$ à $h$ représentent les déformations linéaires et angulaires :

$$
\left.\begin{array}{lll}
a=\frac{\partial u}{\partial x} & b=\frac{\partial v}{\partial r} & c=\frac{\partial w}{r \partial \theta}+\frac{v}{r} \\
f=\frac{\partial v}{r \partial \theta}+\frac{\partial w}{\partial r}-\frac{w}{r} & g=\frac{\partial w}{\partial x}+\frac{\partial u}{r \partial \theta} & h=\frac{\partial u}{\partial r}+\frac{\partial v}{\partial x}
\end{array}\right\}
$$


L'introduction de ces tensions dans les équations du mouvement eut pour résultat celles qu'on appelle les équations de Navier-Stokes :

$$
\begin{gathered}
\rho\left(\frac{\partial u}{\partial t}+u \frac{\partial u}{\partial x}+v \frac{\partial u}{\partial r}+w \frac{\partial u}{r \partial \theta}\right)=-\frac{\partial}{\partial x}(p+\rho \Omega)+\mu\left(\frac{\partial^{2} u}{\partial x^{2}}+\frac{\partial^{2} u}{\partial r^{2}}+\frac{1}{r} \frac{\partial u}{\partial r}+\frac{\partial^{2} u}{r^{2} \partial \theta^{2}}\right) \\
\rho\left(\frac{\partial v}{\partial t}+u \frac{\partial v}{\partial x}+v \frac{\partial v}{\partial r}+w \frac{\partial v}{r \partial \theta}-\frac{w^{2}}{r}\right) \\
=-\frac{\partial}{\partial r}(p+\rho \Omega)+\mu\left(\frac{\partial^{2} v}{\partial x^{2}}+\frac{\partial^{2} v}{\partial r^{2}}+\frac{1}{r} \frac{\partial v}{\partial r}-\frac{v}{r^{2}}+\frac{\partial^{2} v}{r^{2} \partial \theta^{2}}-\frac{2}{r} \frac{\partial w}{r \partial \theta}\right) \\
\rho\left(\frac{\partial w}{\partial t}+u \frac{\partial w}{\partial x}+v \frac{\partial w}{\partial r}+w \frac{\partial w}{r \partial \theta}+\frac{v w}{r}\right) \\
=-\frac{\partial}{r \partial \theta}(p+\rho \Omega)+\mu\left(\frac{\partial^{2} w}{\partial x^{2}}+\frac{\partial^{2} w}{\partial r^{2}}+\frac{1}{r} \frac{\partial w}{\partial r}-\frac{w}{r^{2}}+\frac{\partial^{2} w}{r^{2} \partial 0^{2}}+\frac{2}{r} \frac{\partial v}{r \partial \theta}\right)
\end{gathered}
$$

Si chacune de ces équations était multipliée par la composante respective de la vitesse et si les trois étaient additionnées, le membre de gauche de l'équation résultante représenterait, par unité de volume, la variation temporelle de l'énergie cinétique du fluide, et le membre de droite le travail correspondant. L'énergie est alors conservée. Le travail total, en revanche, se calcule en multipliant la tension sur chaque face d'un élément typique par la composante correspondante de la vitesse, en additionnant les produits algébriquement, et en divisant par le volume de l'élément. Si le travail conservatif est alors soustrait du travail total, le résultat sera la dissipation de l'énergie sous l'action de la viscosité [23]:

$$
\begin{gathered}
\Phi=\mu\left(2 a^{2}+2 b^{2}+2 c^{2}+f^{2}+g^{2}+h^{2}\right) \\
=\left[\left(\frac{\partial u}{\partial x}\right)^{2}+\left(\frac{\partial v}{\partial x}\right)^{2}+\left(\frac{\partial w}{\partial x}\right)^{2}+\left(\frac{\partial u}{\partial r}\right)^{2}+\left(\frac{\partial v}{\partial r}\right)^{2}+\left(\frac{\partial u}{\partial r}\right)^{2}+\left(\frac{\partial u}{r \partial \theta}\right)^{2}+\left(\frac{\partial v}{r \partial \theta}\right)^{2}+\left(\frac{\partial w}{r \partial \theta}\right)^{2-}\right]
\end{gathered}
$$

Reynolds adapta les équations de Stokes à l'étude de l'écoulement turbulent en introduisant une valeur moyenne et la déviation instantanée de la moyenne, pour les trois composantes de la vitesse, et pour la pression, et en éliminant dans la suite tous les termes dont la valeur temporelle moyenne était nécessairement égale à zéro. Si on indique comme d'habitude les valeurs moyennes par des tirets supérieurs et les déviations par des « primes », et si, pour raison de simplification, on réduit les équations à celles qui s'appliquent aux problèmes de l'écouJement moyen stationnaire avec $w=0$ dans la direction $\theta$, les équations de Reynolds deviendront:

$$
\begin{aligned}
& \rho\left(\bar{u} \frac{\partial \bar{u}}{\partial x}+\bar{v} \frac{\partial \bar{u}}{\partial r}+\overline{u^{\prime} \frac{\partial u^{\prime}}{\partial x}}+\overline{v^{\prime} \frac{\partial u^{\prime}}{\partial r}}+\overline{w^{\prime} \frac{\partial u^{\prime}}{r \partial \theta}}\right)=-\frac{\partial}{\partial x}(\bar{p}+\rho \Omega)+\mu\left(\frac{\partial^{2} \bar{u}}{\partial x^{2}}+\frac{\partial^{2} \bar{u}}{\partial r^{2}}+\frac{1}{r^{2}} \frac{\partial \bar{u}}{\partial r}\right) \\
& \left.\rho\left(\bar{u} \frac{\partial \bar{v}}{\partial x}+\bar{v} \frac{\partial \bar{v}}{\partial r}+\overline{u^{\prime} \frac{\partial v^{\prime}}{\partial x}}+\overline{v^{\prime} \frac{\partial v^{\prime}}{\partial r}}+\overline{w^{\prime} \frac{\partial v^{\prime}}{r \partial \theta}}-\overline{\frac{w^{\prime 2}}{r}}\right)=-\frac{\partial}{\partial r}(\bar{p}+e \Omega)+\mu\left(\frac{\partial^{2} \bar{v}}{\partial x^{2}}+\frac{\partial^{2} \bar{v}}{\partial r^{2}}+\frac{1}{r} \frac{\partial^{2} \bar{v}}{\partial r}-\frac{\bar{u}}{r^{2}}\right)\right)
\end{aligned}
$$

L’équation de continuité correspondante est simplement :

$$
\frac{\partial(r \bar{u})}{\partial x}+\frac{\partial(r \vec{v})}{\partial r}=0
$$

Au premier coup d'œil, les équations sembleraient être changées seulenient par le remplacement des valeurs instantanées par les valeurs moyennes, et par l'addition de quelques termes renfermant des combinaisons moyennes des variations. Les membres de droite de ces équations ont ainsi été simplifiés par l'élimination des tensions fluctuantes visqueuses, qui — si compliquées qu'elles soient - ont nécessairement une valeur temporelle moyenne de zéro. En même temps, pourtant, les termes « accélérateur's 》 variables, qui n'ont pas nécessairement une valeur moyenne de zéro, ont rendu les membres de gauche d'autant plus compliqués. En effet, ces termes accélérateurs peuvent se construire comme transferts moyens de quantités de mouvement, et par conséquent (par analogie avec la théorie de la viscosité moléculaire) comme tensions dues au mélange d'une échelle molaire au lieu de moléculaire. Cela s'éclaircit au cours de la manipulation suivante des quantités en question : 


$$
\left.\begin{array}{r}
\boldsymbol{u}^{\prime} \frac{\partial u^{\prime}}{\partial x}+v^{\prime} \frac{\partial u^{\prime}}{\partial r}+\boldsymbol{v}^{\prime} \frac{\partial u^{\prime}}{r \partial \theta}=\frac{\partial\left(\boldsymbol{u}^{\prime} u^{\prime}\right)}{\partial x}+\frac{\partial\left(u^{\prime} v^{\prime}\right)}{\partial r}+\frac{\partial\left(u^{\prime} w^{\prime}\right)}{r \partial \theta}-u^{\prime}\left(\frac{\partial u^{\prime}}{\partial x}+\frac{\partial v^{\prime}}{\partial r}+\frac{v^{\prime}}{r}+\frac{\partial w^{\prime}}{r \partial \theta}\right)+u^{\prime} \frac{v^{\prime}}{r} \\
u^{\prime} \frac{\partial v^{\prime}}{\partial x}+v^{\prime} \frac{\partial v^{\prime}}{\partial r}+w^{\prime} \frac{\partial v^{\prime}}{r \partial \theta}-\frac{w^{\prime 2}}{r}=\frac{\partial\left(u^{\prime} v^{\prime}\right)}{\partial x}+\frac{\partial\left(v^{\prime} v^{\prime}\right)}{\partial r}+\frac{\partial\left(v^{\prime} w^{\prime}\right)}{r \partial \theta} \\
-v^{\prime}\left(\frac{\partial u^{\prime}}{\partial x}+\frac{\partial v^{\prime}}{\partial r}+\frac{v^{\prime}}{r}+\frac{\partial w^{\prime}}{r \partial \theta}\right)+v^{\prime} \frac{v^{\prime}}{r}+\frac{w^{\prime} w^{\prime}}{r}
\end{array}\right\}
$$

Les termes à l'intérieur des grandes parenthèses sont égaux à zéro, en vertu de l'équation de continuité, et les produits de e par le restant des termes - logiquement nommés tensions de Reynolds seront tous trouvés analogues aux tensions de Stokes exprimées en fonction de la vitesse moyenne. En d'autres mots :

$$
\left.\begin{array}{c}
\bar{\sigma}_{x}=-p+2 \mu \frac{\partial \bar{u}}{\partial x}-\overline{\rho u^{\prime 2}} ; \quad \bar{\sigma}_{r}=-p+2 \mu \frac{\partial \bar{v}}{\partial r}-\rho \overline{v^{\prime 2}} ; \quad \overline{\sigma_{\theta}}=-p+2 \mu \frac{\bar{v}}{r}-\rho \overline{w^{\prime 2}} \\
\bar{\tau}_{r \theta}=0 ; \quad \bar{\tau}_{\theta x}=0 ; \bar{\tau}_{x r}=\bar{\tau}_{r x}=\bar{\tau}=\mu\left(\frac{\partial \bar{u}}{\partial r}+\frac{\partial \bar{v}}{\partial x}\right)-\rho \overline{u^{\prime} v^{\prime}}
\end{array}\right\}
$$

L'introduction de ces tensions dans les équations de Reynolds, avec une manipulation des termes moyens de la gauche, se ramène à la forme assez symétrique :

$$
\left.\begin{array}{l}
-\frac{\rho}{r}\left[\frac{\partial\left(\boldsymbol{r} \bar{u}^{2}\right)}{\partial x}+\frac{\partial(r \bar{u} \bar{v})}{\partial r}\right]=-\rho \frac{\partial \Omega}{\partial x}+\frac{1}{r}\left[\frac{\partial\left(r \overline{\sigma_{x}}\right)}{\partial x}+\frac{\partial(r \bar{\tau})}{\partial r}\right] \\
\frac{\rho}{r}\left[\frac{\partial(r \bar{u} \bar{u})}{\partial x}+\frac{\partial\left(r \bar{v}^{2}\right)}{\partial r}\right]=-\rho \frac{\partial \Omega}{\partial r}+\frac{1}{r}\left[\frac{\partial(r \bar{\tau})}{\partial x}+\frac{\partial\left(r \overline{\sigma_{r}}\right)}{\partial r}-\bar{\sigma}_{\theta}\right]
\end{array}\right\}
$$

Des équations d'accélération comme celles-ci peuvent aussi être interprétées comme des relations entre la variation de quantité du mouvement, à gauche, et l'impulsion correspondante, à droite. N'importe laquelle d'entre elles peut ainsi être intégrée sur une zone donnée de l'espace pour produire une équation d'impulsion du type fréquemment rencontré en hydraulique. L'intégrale volumétrique de l'équation de Reynolds pour la direction axiale sera évidemment :

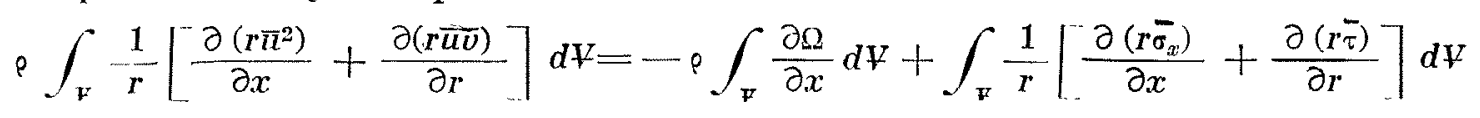

En appliquant la relation de Gauss :

$$
\int_{v} \frac{1}{r}\left[\frac{\partial(r P)}{\partial x}+\frac{\partial(r Q)}{\partial r}\right] d \psi=\int_{S}\left(P \frac{\partial x}{\partial n}+Q \frac{\partial r}{\partial n}\right) d S
$$

dans laquelle $n$ représente la distance perpendiculaire hors de la surface, les intégrales volumétriques convenables peuvent être transformées en intégrales superficielles, avec le résultat significatif que :

$$
\rho \int_{S}\left(\bar{u}^{2} \frac{\partial x}{\partial n}+\bar{u} \bar{v} \frac{\partial r}{\partial n}\right) d S=-\vartheta \int_{\nabla} \frac{\partial \Omega}{\partial x} d \psi+\int_{S}\left(\vec{\sigma}_{x} \frac{\partial x}{\partial n}+\bar{\tau} \frac{\partial r}{\partial n}\right) d S
$$

Le membre de gauche représente alors la variation de la composante $x$ de la quantité de mouvement par unité de volume pendant que le fluide passe par la zone en question - c'est-à-dire la différence entre la quantité sortante et la quantité entrante par unité de temps. En général, le fluide entre ou sort de la zone par tous les points de la surface environnante; cependant, en hydraulique, la zone considérée est ordinairement limitée sur les côtés par des parois solides, au travers desquelles aucun fluide ne s'écoule, et, aux extrémités, par des sections normales, auxquelles, seule, une des composantes de la vitesse s'applique. Le terme gravitationnel est simplement la composante exacte de la pesanteur du fluide contenu dans la zone. Toutes les tensions internes sont évidemment sans effet (i.e. contrebalancées). Pour le reste des tensions, la partie moyenne visqueuse est limitée en général à l'action tangentielle le long des parois, dont les tensions moyennes turbulentes disparaissent; de même, la partie moyenne turbulente est limitée en gẻnéral aux sections normales de l'écoulement, où les tensions visqueuses sont, en comparaison, négligeables. 


\section{Equations d'énergie}

Puisque les relations comme celles de l'accélération ou de la quantité de mouvement s'écrivent nécessairement en fonction de composantes vectorielles, elles ne peuvent pas comprendre une mesure directe de la perte d'énergie, qui est une quantité scalaire. De même que pour les équations de l'écoulement visqueux, afin d'obtenir des expressions pour l'énergie de l'écoulement turbulent [25], il faut multiplier chaque équation par la composante respective de la vitesse (écrite partout comme la somme des parties moyennes et variables) et après, additionner les résultats. (On doit remarquer que, pour retenir tous les termes importants, il faut effectuer la multiplication avant de faire les moyennes temporelles et d'omettre des termes.) L’issue finale moyenne est la variation temporelle totale de l'énergie par unité de volume :

$$
\begin{aligned}
& \frac{\rho}{2}\left(\bar{u} \frac{\partial \overline{V^{2}}}{\partial x}+\bar{v} \frac{\partial \overline{V^{2}}}{\partial r}+\bar{u} \frac{\partial \overline{V^{\prime 2}}}{\partial x}+\bar{v} \frac{\partial \overline{V^{\prime 2}}}{\partial r^{\prime}}+\overline{u^{\prime} \frac{\partial V^{\prime 2}}{\partial x}}+\overline{v^{\prime} \frac{\partial V^{\prime 2}}{\partial r}}+\overline{w^{\prime} \frac{\partial V^{\prime 2}}{r^{\prime} \partial}}\right)
\end{aligned}
$$

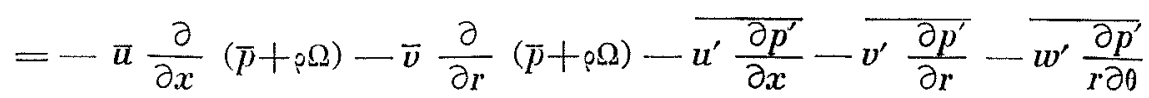

$$
\begin{aligned}
& +\mu\left(\bar{u} \frac{\partial^{2} \bar{u}}{\partial x^{2}}+\bar{u} \frac{\partial^{2} \bar{u}}{\partial r^{2}}+\frac{\bar{u}}{r} \frac{\partial \bar{u}}{\partial r}+\bar{v} \frac{\partial^{2} \bar{v}}{\partial x^{2}}+\bar{v} \frac{\partial^{2} \bar{v}}{\partial r^{2}}+\frac{\bar{v}}{r} \frac{\partial \bar{v}}{\partial r}-\frac{\bar{v}^{2}}{r^{2}}\right. \\
& +\overline{u^{\prime} \frac{\partial^{2} u^{\prime}}{\partial x^{2}}}+\overline{\overline{u^{\prime}}} \overline{\frac{\partial^{2} u^{\prime}}{\partial r^{2}}}+\overline{\frac{u^{\prime}}{r} \frac{\partial u^{\prime}}{\partial r}}+\overline{u^{\prime} \frac{\partial^{2} u^{\prime}}{r^{2} \partial \theta^{2}}} \\
& +\overline{+v^{\prime} \frac{\partial^{2} v^{\prime}}{\partial x^{2}}}+\overline{v^{\prime} \frac{\partial^{2} v^{\prime}}{\partial r^{2}}}+\overline{\overline{v^{\prime}}} \frac{\partial v^{\prime}}{\partial r}+\overline{+v^{\prime} \frac{\partial^{2} v^{\prime}}{r^{2} \partial \theta^{2}}}-\overline{2 v^{\prime} \frac{\partial w^{\prime}}{r \partial \theta}}-\overline{\frac{v^{2}}{r^{2}}} \\
& \left.+\overline{w^{\prime}} \frac{\partial^{2} w^{\prime}}{\partial x^{2}}+\overline{w^{\prime} \frac{\partial^{2} w^{\prime}}{\partial r^{2}}}+\overline{\frac{w^{\prime}}{r} \frac{\partial w^{\prime}}{\partial r}}+\overline{w^{\prime} \frac{\partial^{2} w^{\prime}}{r^{2} \partial \theta^{2}}}+\overline{2 w^{\prime} \frac{\partial v^{\prime}}{r \partial \theta}}-\overline{\frac{w^{\prime 2}}{r^{2}}}\right) \\
& -\rho\left(\bar{u} \frac{\partial \overline{u^{\prime 2}}}{\partial x}+\bar{u} \frac{\partial \overline{u^{\prime} \overline{v^{\prime}}}}{\partial r}+\bar{u} \frac{\overline{u^{\prime} v^{\prime}}}{r}+\bar{v} \frac{\partial \overline{u^{\prime} v^{\prime}}}{\partial x}+\bar{v} \frac{\partial \overline{v^{\prime 2}}}{\partial r}+\bar{v} \frac{\overline{v^{\prime 2}}}{r}\right. \\
& \left.+\dot{u^{\prime 2}} \frac{\partial \bar{u}}{\partial x}+\overline{u^{\prime} v^{\prime}} \frac{\partial \bar{u}}{\partial r^{\prime}}+\overline{u^{\prime} v^{\prime}} \frac{\partial \bar{v}}{\partial x}+\overline{v^{\prime 2}} \frac{\partial \bar{v}}{\partial r}\right)
\end{aligned}
$$

Si les équations de Reynolds avaient été multipliées par les composantes respectives de la vitesse et ensuite additionnées, les termes pourraient être arrangés pour obtenir la forme suivante de l'équation d'énergie pour le mouvement moyen tout seul :

$$
\begin{aligned}
& -\rho \bar{u} \frac{\partial \Omega}{\partial x}-\rho \bar{\nu} \frac{\partial \Omega}{\partial r}+\frac{1}{r}\left[\frac{\partial}{\partial x} r\left(\bar{u} \bar{\sigma}_{s}+\bar{v} \bar{\tau}\right)+\frac{\partial}{\partial r} r\left(\bar{u} \bar{\tau}+\bar{v} \bar{\sigma}_{r}\right)\right] \\
& =\frac{\rho}{2}\left(\bar{u} \frac{\partial \bar{V}^{2}}{\partial x}+\bar{v} \frac{\partial \bar{V}^{2}}{\partial r}\right)+\bar{\sigma}_{r} \frac{\partial \bar{u}}{\partial x}+\bar{\sigma}_{r} \frac{\partial \bar{v}}{\partial r}+\bar{\sigma}_{\theta} \frac{\bar{u}}{r}+\bar{\tau}\left(\frac{\partial \bar{u}}{\partial r}+\frac{\partial \bar{u}}{\partial x}\right)
\end{aligned}
$$

En fonction des tensions individuelles, ceci devient:

$$
\begin{aligned}
& -u \frac{\partial}{\partial x}(\bar{p}+\rho \Omega)-\bar{v} \frac{\partial}{\partial r}(\bar{p}+\rho \Omega) \\
& +\frac{\mu}{r}\left\{\frac{\partial}{\partial x} r\left[2 \bar{u} \frac{\partial \bar{u}}{\partial x}+\bar{v}\left(\frac{\partial \bar{u}}{\partial r}+\frac{\partial \bar{v}}{\partial x}\right)\right]+\frac{\partial}{\partial r} r\left[\bar{u}\left(\frac{\partial \bar{u}}{\partial r}+\frac{\partial \bar{v}}{\partial x}\right)+2 \bar{v} \frac{\partial \bar{v}}{\partial r}\right]\right\} \\
& -\frac{\rho}{r}\left[\frac{\partial}{\partial x} r\left(\bar{u} \overline{u^{\prime 2}}+\bar{v} \overline{u^{\prime} v^{\prime}}\right)+\frac{\partial}{\partial r} r\left(\bar{u} \overline{u^{\prime} v^{\prime}}+\bar{v} \overline{v^{2}}\right)\right] \\
& =\frac{\rho}{2}\left(\bar{u} \frac{\partial \bar{V}^{2}}{\partial x}+\bar{v} \frac{\partial \bar{V}^{2}}{\partial r}\right)+\mu\left[2\left(\frac{\partial \bar{u}}{\partial x}\right)^{2}+2\left(\frac{\partial \bar{v}}{\partial r}\right)^{2}+2\left(\frac{\bar{v}}{r}\right)^{2}+\left(\frac{\partial \bar{u}}{\partial r}+\frac{\partial \bar{v}}{\partial x}\right)^{2}\right] \\
& -\rho\left[\overline{u^{\prime 2}} \frac{\partial \bar{u}}{\partial x}+\overline{v^{\prime 2}} \frac{\partial \bar{v}}{\partial r}+\overline{w^{\prime 2}} \frac{\bar{v}}{r}+\overline{u^{\prime} v^{\prime}}\left(\frac{\partial \bar{u}}{\partial r}+\frac{\partial \bar{v}}{\partial x}\right)\right]
\end{aligned}
$$


dont la soustraction de l'équation pour la variation totale donne l'équation de l'énergie pour le mouvement turbulent tout seul :

$$
\begin{aligned}
& -\overline{u^{\prime} \frac{\partial p^{\prime}}{\partial x}}-\overline{v^{\prime} \frac{\partial p^{\prime}}{\partial r^{\prime}}}-\overline{w^{\prime} \frac{\partial p^{\prime}}{r^{\prime} \partial 0}}-\rho\left[\overline{u^{\prime 2}} \frac{\partial \bar{u}}{\partial x}+\overline{v^{\prime 2}} \frac{\partial \bar{v}}{\partial r}+\overline{w^{\prime 2}} \frac{\bar{v}}{r}+\overline{u^{\prime} y^{\prime}}\left(\frac{\partial \bar{u}}{\partial r}+\frac{\partial \bar{v}}{\partial x}\right)\right] \\
& -\frac{\mu}{r}\left\{\frac { \partial } { \partial x } r \left[\overline{u^{\prime} \frac{\partial u^{\prime}}{\partial x}}+\overline{\left.v^{\prime}\left(\frac{\partial u^{\prime}}{\partial r}+\frac{\partial v^{\prime}}{\partial x}\right)+\overline{w^{\prime}\left(\frac{\partial w^{\prime}}{\partial x}+\frac{\partial u^{\prime}}{r \partial \theta}\right)}\right]}\right.\right. \\
& +\frac{\partial}{\partial r} r\left[\overline{u^{\prime}\left(\frac{\partial u^{\prime}}{\partial r}+\frac{\partial v^{\prime}}{\partial x}\right)}+\overline{v^{\prime} \frac{\partial v^{\prime}}{\partial r}}+\overline{w^{\prime}\left(\frac{\partial v^{\prime}}{r^{\prime} \partial \theta}+\frac{\partial w^{\prime}}{\partial r}-\frac{w^{\prime}}{r}\right)}\right] \\
& \left.+\frac{\partial}{r \partial \theta} r\left[\overline{u^{\prime}\left(\frac{\partial w^{\prime}}{\partial x}+\frac{\partial u^{\prime}}{r \partial \theta}\right)}+\overline{v^{\prime}\left(\frac{\partial v^{\prime}}{r \partial \theta}+\frac{\partial w^{\prime}}{\partial r}-\frac{w^{\prime}}{r}\right)}+\overline{w^{\prime}\left(\frac{\partial w^{\prime}}{r \partial \theta}+\frac{v^{\prime}}{r}\right)}\right]\right\} \\
& =\frac{p}{2}\left(\bar{u} \frac{\partial \overline{V^{\prime 2}}}{\partial x}+\bar{v} \frac{\partial \overline{V^{\prime 2}}}{\partial r}+\overline{u^{\prime} \frac{\partial V^{\prime 2}}{\partial x}}+\overline{v^{\prime} \frac{\partial V^{\prime 2}}{\partial r}}+\overline{w^{\prime} \frac{\partial V^{\prime 2}}{r \partial \theta}}\right) \\
& +\kappa\left[\left(\overline{\left.\frac{\partial u^{\prime}}{\partial x}\right)^{2}}+\left(\overline{\left.\frac{\partial v^{\prime}}{\partial x}\right)^{2}}+\left(\overline{\left.\frac{\partial w^{\prime}}{\partial x}\right)^{2}}+\left(\overline{\left.\frac{\partial u^{\prime}}{\partial r}\right)^{2}}+\left(\overline{\left.\frac{\partial v^{\prime}}{\partial r}\right)^{2}}+\left(\overline{\left.\frac{\partial w^{\prime}}{\partial r}\right)^{2}}+\left(\overline{\left.\frac{\partial u^{\prime}}{r \partial \theta}\right)^{2}}+\left(\frac{\overline{\partial v^{\prime}}}{r \partial \theta}\right)^{2}+\left(\frac{\partial w^{\prime}}{r \partial \theta}\right)^{2}\right]\right.\right.\right.\right.\right.\right.\right.
\end{aligned}
$$

L'interprétation réfléchie des divers termes de ces équations est d'une très grande importance. Les trois dernières ont été arrangées de telle sorte que les membres de gauche représentent le travail et les membres de droite la variation d'énergie correspondante. Les termes qui indiquent le travail fait par la pression et par la gravité sont évidents, de même que les variations de l'énergie cinétique moyenne et turbulente $\rho \bar{V}^{2}$ et $\rho \bar{V}^{\prime 2} / 2$ par suite de la convection par le mouvement moyen et de la diffusion par la turbulence. Exactement comme dans le cas de l'écoulement purement visqueux déjà discuté brièvement, le travail fait par les tensions dans l'écoulement turbulent est en partie conservateur et en partie dissipateur. En analogie avec l'analyse précédente de l'écoulement visqueux, les termes conservateurs venant des équations de Reynolds ont été remplacés par la différence entre les termes totaux et les termes dissipateurs et mis à part comme dans la première équation pour l'écoulement moyen - totaux à gauche et dissipateurs à droite. Mais les tensions consistent maintenant dans les composantes visqueuses et turbulentes, comme décrites dans l'équation alternative de l'écoulement moyen. La signification de la partie dissipatrice visqueuse est telle qu'auparavant. Pourtant, la partie comprenant ce qui est réellement des termes « accélérateurs » dus à la variation turbulente, ne peut pas être vraiment dissipatrice, parce qu'elle ne dépend pas de la viscosité; néanmoins elle est décidément non conservatrice en ce qui concerne le mouvement moyen. En d'autres termes, la quantité :

$$
-\rho\left[\overline{u^{\prime 2}} \frac{\partial \bar{u}}{\partial x}+\overline{v^{2}} \frac{\partial \bar{v}}{\partial r}+\overline{w^{\prime 2}} \frac{\bar{v}}{r}+\overline{u^{\prime} v^{\prime}}\left(\frac{\partial \vec{u}}{\partial \vec{r}}+\frac{\partial \bar{v}}{\partial x}\right)\right]
$$

représente la rapidité avec laquelle l'énergie est perdue irrécouvrablement par le mouvernent moyen et gagnée par la turbulence.

Ces termes pour la production de lurbulence paraissent, encore une fois, dans l'équation de l'énergie pour le mouvement turbulent, mais à gauche, car ils représentent maintenant une fourniture extérieure d'énergie, quelque peu comme le travail fait par des forces externes. Du travail est fait encore par les tensions visqueuses aussi bien que par celles du mouvement secondaire. Enfin, tout à droite, se trouvenl les termes représentant la dissipation visqueuse dans la turbulence, laquelle est de beaucoup' la partic majeure de la dissipation.

L'équation d'énergie, comme les trois composantes de l'équation de la quantité de mouvement, devient pratiquement utile lorsqu'elle est intégrée sur la zone considérée. Pour le moment, cette opération sera indiquée schématiquement sous la forme :

$$
\begin{aligned}
& -\cdots \int_{\bar{v}}\left(\bar{u} \frac{\partial \Omega}{\partial x}+\bar{v} \frac{\partial \Omega}{\partial r}\right) d \Psi+\int_{v} \frac{1}{r}\left[\frac{\partial}{\partial x} r\left(\overline{u \sigma_{x}}+\overline{v \tau_{x r}}+\overline{w \tau_{x \theta}}\right)\right. \\
& \left.+\frac{\partial}{\partial r} r\left(\overline{u \tau}_{r w}+\overline{v \sigma}_{r}+\bar{w}_{r \theta}\right)+\frac{\partial}{r \partial \theta} r\left(\overline{u \tau}_{\theta x}+\overline{v \tau}_{\theta r}+\overline{w \sigma}_{\theta}\right)\right] d \Psi \\
& =\frac{\rho}{2} \int_{F}\left(\overline{u \frac{\partial V^{2}}{\partial x}}+\overline{v \frac{\partial V^{2}}{\partial r}}+\overline{w \frac{\partial V^{2}}{r \partial \theta}}\right) d \forall+\int_{\nabla}\left[\overline{\sigma_{x} \frac{\partial u}{\partial x}}+\overline{\sigma_{r} \frac{\partial v}{\partial r}}+\overline{\sigma_{\theta} \frac{\partial w}{r \partial \theta}}\right. \\
& +\overline{+\tau_{r \theta}\left(\frac{\partial v}{r \partial \theta}+\frac{\partial w}{\partial r}-\frac{w}{r}\right)}+\overline{\tau_{\theta w}\left(\frac{\partial w}{\partial x}+\frac{\partial u}{r \partial \theta}\right)}+\overline{\left.\tau_{x r}\left(\frac{\partial u}{\partial r}+\frac{\partial v}{\partial x}\right)\right]} d \Psi
\end{aligned}
$$


qui, en appliquant la transformation de Gauss entre les intégrales spaciales et superficielles, s'écrit dans une manière plus significative telle que :

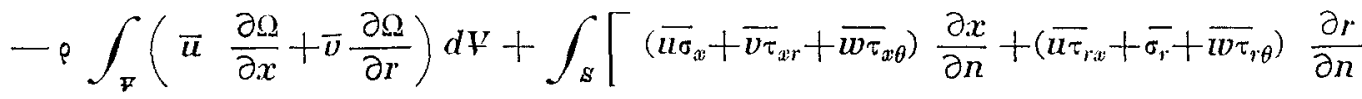

$$
\begin{aligned}
& \left.+\left(\overline{u \tau}_{\theta \theta}+\overline{v \tau}_{\theta r}+\overline{w \sigma}_{\theta}\right) \frac{r \partial \theta}{\partial n}\right] d S=\frac{\rho}{2} \int_{S}\left(\overline{u V^{2}} \frac{\partial x}{\partial n}+\overline{v V^{2}} \frac{\partial r}{\partial n}+\overline{w V^{2}} \frac{r \partial \theta}{\partial n}\right) d S \\
& +\int_{r}\left[\overline{\sigma_{x} \frac{\partial u}{\partial x}}+\overline{\sigma_{r} \frac{\partial v}{\partial r}}+\overline{\sigma_{\theta} \frac{\partial w}{r \partial \theta}}+\overline{\tau_{r \theta}\left(\frac{\partial v}{r \partial \theta}+\frac{\partial w}{\partial r}-\frac{w}{r}\right)}+\overline{\left.\tau_{\theta x}\left(\frac{\partial w}{\partial x}+\frac{\partial u}{r \partial \theta}\right)+\tau_{a r}\left(\frac{\partial u}{\partial r}+\frac{\partial v}{\partial x}\right)\right] d \psi}\right.
\end{aligned}
$$

Ici, les termes successifs ont à peu près la même signification qu'auparavant, travail à gauche et transformation d'énergie à droite. Pour évaluer les termes, il faut se souvenir que chaque composante de la vitesse consiste en une partie moyenne et une partie variable, que chaque tension consiste en une partie visqueuse et une partie due à l'inertie, et que tous les produits qui ne sont pas nécessairement égaux à zéro ont leur place dans l'expression qui en résulte.

\section{Interprétation du point de vue de l'analyse isotrope}

Les relations précédentes indiquent évidemment un état d'équilibre entre la production, le transfert, et la dissipation de l'énergie turbulente: production aux dépens de l'écoulement moyen, transfert par convection et diffusion, et dissipation au moyen de la viscosité. Dans chaque série d'action, les composantes moyennes et variables de la vitesse jouent manifestement un rôle primaire, mais la seule indication de l'échelle de l'action se trouve dans les dérivées, en fonction de l'espace, des composantes et de leurs produits. Une évaluation quantitative de cette échelle n'est possible qu'au moyen d'une analyse statistique des composantes de la vitesse comme fonction de l'espace ou du temps.

Chaque composante de la vitesse, à un point quelconque d'un écoulement turbulent, varie généralement avec le temps dans une manière continue mais qui ne peut pas être autrement pré-

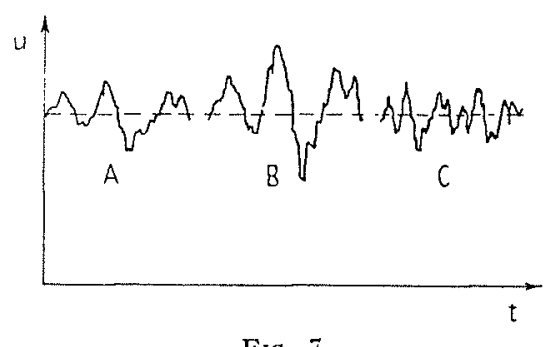

Fig. 7

Enregistrements temporels des fluctuations de vitesse Temporal records of velocity fluctuation

dite, comme l'indique la figure 7 . Si $f(u)$ représente la fréquence avec laquelle la composante $u$ se trouve entre les valeurs $u$ et $u+d u$, de telle sorte que :

$$
\int_{0}^{\infty} f(u) d u=1
$$

alors la répartition de la fréquence de la variation aura en gros une forme de la fonction de probabilité montrée par la figure 8. La valeur

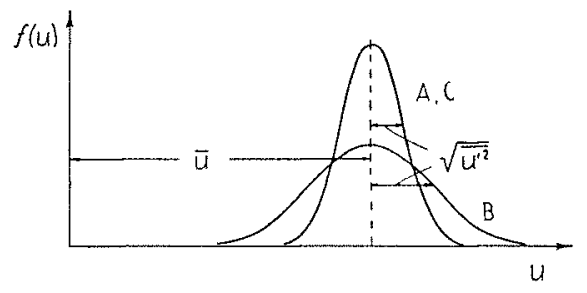

FIG. 8

Diagrammes de fréquence des fluctuations de vitesse Frequency diagrams of velocity fluctuation

moyenne de la composante correspond à l'abscisse du centroïde de la surface délimitée par la courbe de fréquence :

$$
\bar{u}=\int_{0}^{\infty} u f(u) d u
$$

et la racine du carré moyen de la déviation à partir de celte moyenne est représentée par le rayon de giration de la même surface de fréquence autour de son axe centroïdal :

$$
\sqrt{\overline{u^{2}}}=\left[\int_{0}^{\infty}(u-\bar{u})^{2} f(u) d u\right]^{1 / 2}
$$

De cette façon, tandis que les traces $A$ et $B$ de la figure 7 ont la même valeur moyenne, les racines des carrés moyens de leurs déviations diffèrent considérablement.

La trace $\mathrm{C}$ a évidemment la même moyenne que $A$ et $B$ et la même racine du moyen carré de la déviation que $A$, mais elle diffère de tous les deux d'une manière qu'il faut caractériser par références aux abscisses plutôt qu'aux ordonnées, c'est-à-dire, en déterminant la corrélation moyenne $R_{t}$ entre deux valeurs de la distance $\Delta t$ 
variable, séparées sur l'enregistrement:

$$
R_{l}=\frac{\overline{u^{\prime}(t) u^{\prime}(t+\Delta t)}}{\overline{u^{\prime 2}}}
$$

Pendant que $\Delta t$ tend vers zéro, $R_{t}$ tend nécessairement vers l'unité (fig. 9), mais en revan-

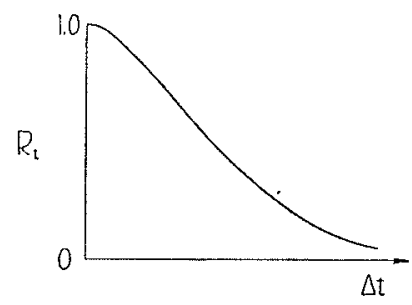

FiG. 9

Corrélation temporelle des fluctuations

Time correlation of velocity fluctuations

che $R_{t}$ tend vers zéro lorsque $\Delta t$ excède une certaine valeur. Si la déviation est petite par rapport à la moyenne, le temps $\Delta t$ peut être changé avec une bonne approximation en une distance axiale correspondante :

$$
\Delta x \approx \bar{u} \Delta t
$$

Avec cela, le diagramme de la corrélation $R_{x}$ (fig. 10) devient l'indicatif de l'échelle linéaire

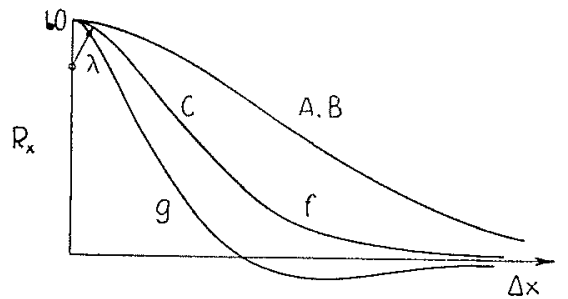

Fig. 10

Corrélations spatiales des fluctuations

Space correlations of velocity flucturtions

du mouvement secondaire. Soit que le point où la corrélation s'approche de zéro, soit que, préfćrablement, l'aire de la surface sous le diagramme, s'utilisent comme mesure de cette longueur, on voit que les traces $A$ et $B$ sont caractérisées par une échelle qui est sensiblement plus grande que celle de $C$.

Si l'on imagine les variations de la vitesse dans l'écoulement turbulent comme étant le résultat d'un système hétérogène de tourbillons transportés par le courant moyen, l'échelle linéaire ensuite obtenue de la fonction de corrélation, peut être considérée conme représentant la grandeur moyenne des tourbillons. Que la notion de tourbillon ne soit pas déraisonnable est indiqué par la forme de la fonction de corrélation pour les composantes de la vitesse normale à la ligne entre les points considérés, ce qui est illustré par la courbe $g$ de la figure 10 ; c'est-à-dire que la fonction devient négalive pour des distances un peu plus petites que celles auxquelles la courbe $f$ s'approche de zéro, parce que, en effet, le fluide sur l'un des côtés du tourbillon a tendance à se déplacer en amont pendant que celui de l'autre côté se déplace en aval.

Semblable aux composantes de la vitesse, la fonction de corrélation doit varier avec la direction, parce qu'elle dépend de l'orientation de chacune des deux composantes et de celle de la ligne entre les deux — c'est-à-dire le vecteur de séparation $s$. Une telle double corrélation est, par le fait, une fonction tensorielle avec neuf composantes différentes, dont toutes sont liées à l'échelle des tourbillons. (On doit noter que les tensions de Reynolds sont les valeurs limites de certaines de ces composantes, sous la condition d'une séparation égale à zéro, de telle sorte qu'elles ont perdu leur signification linéaire.) Chaque composante est nécessairement une quantité qui par elle-même ne donne aucune indication de la véritable répartition de grandeur des tourbillons.

Une telle connaissance s'obtient de la même manière que pour résoudre un enregistremenl de vitesse tel que celui de la figure 7 , en une fonction spectrale qui montre la répartition de l'énergie attribuable à la composante de la vilesse donnée, pour toutes les fréquences de variation ou longueurs d'ondes présentes dans l'enregistrement.

Une telle fonction est, de fait, une transformée de Fourier de la fonction de corrélation [26]. Toutefois, le spectre général a la même nature d'un tenseur que la corrélation générale, et c'est le tenseur, au lieu d'une de ses composantes, qui est d'intérêt actuel. Si l'énergie cinétique moyenne de la turbulence par unité de volume était reconnue comme étant le produit de la densité par la moitié de la somme des carrés moyens des variations, alors celle-ci pourrait s'écrire comme l'intégrale de l'énergie par l'unité de nombre d'onde $E(k)$ sur toute la gamme de nombres d'onde :

$$
\left.\frac{\rho}{2} \overline{\left(u^{\prime 2}\right.}+\overline{v^{\prime 2}}+\overline{w^{\prime 2}}\right)=\int_{0}^{\infty} E(k) d k
$$

Ici $k$ est la grandeur du vecteur du nombre d'ondes [27], c'est-à-dire un paramètre tensoriel égal à $2 \pi$ divisés par une sorte de longueur d'onde spatiale. Le point essentiel est que $k$ peut être regardé comme la valeur réciproque d'une longueur qui caractérise l'échelle des tourbillons. Le diagramme d'un tel spectre (fig. 11) représente par conséquent la répartition de l'énergie entre les différentes grandeurs de tourbillons, lesquels 


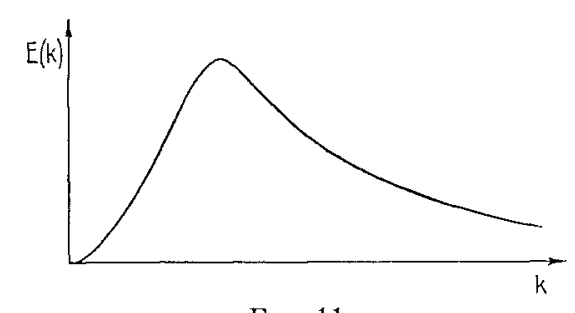

Fra. 11

Répartition spectrale d'énergie turbulente Spectral distribution of turbulent energy

varient d'une très grande dimension près do l'origine jusqu'à une très petite à l'extrêmedroite.

Pour le cas spécial, introduit par Taylor [28], dans lequel la turbulence est non seulement homogène (indépendanto de la position) mais aussi isotrope (indépendante de la direction), les conditions sont simplifiées à tel point que les carrés moyens de la fluctuation sont tous identiques:

$$
\overline{u^{\prime \prime 2}}=\overline{v^{\prime 2}}={\overline{w^{2}}}^{2}
$$

Les carrés moyens de leurs dérivées sont alors deux fois plus petits dans les directions des composantes que dans les directions perpendiculaires :

$$
\begin{aligned}
& \overline{\left(\frac{\partial u^{\prime}}{\partial x}\right)^{2}}=\overline{\left(\frac{\partial v^{\prime}}{\partial r}\right)^{2}}=\overline{\left(\frac{\partial w^{\prime}}{r \partial \theta}\right)^{2}} \\
& =\frac{1}{2}\left(\overline{\left.\frac{\partial r^{\prime}}{\partial r}\right)^{2}}=\frac{1}{2}\left(\overline{\left.\frac{\partial v^{\prime}}{r \partial \theta}\right)^{2}}\right. \text { etc. }\right.
\end{aligned}
$$

el le lenseur pour la double corrélation se réduil à deux fonctions indépendantes [29]:

$$
g=f+\frac{s}{2} \frac{\partial f}{\partial s}
$$

La dissipation de l'énergic est ensuite exprimable en fonction d'une seule dérivée de la vitesse :

$$
\varepsilon=15 \mu \overline{\left(\frac{\partial u^{\prime}}{\partial x}\right)^{2}}
$$

et aussi en fonction du rayon de courbure $\lambda$ de la fonction de corrélation $f$ de la figure 10 à $R=1$ :

$$
\varepsilon=15 \mu \frac{\overline{u^{\prime 2}}}{\lambda^{2}}
$$

La fonction spectrale générale, de plus, peut ètre réduite pour fournir l'expression [25]:

$$
\frac{\partial E(k)}{\partial t}=T(k)-2 \mu k^{2} E(k)
$$

laquelle énonce que la variation de l'énergie d'un nombre d'onde donné est égale au gain net d'énergie au moyen du transfert des plus petits aux plus grands nombres d'onde, moins la dissipation à ce nombre d'onde.

Le transfert d'énergie d'un nombre d'onde à un autre est un effet inertiel, pendant que celui de la dissipation est visqueux, et le rapport entre les deux devrait donc varier avec le nombre de Reynolds de l'écoulement et avec la position relative dans le spectre du nombre d'onde considéré. Néanmoins Kolmogoroff [30] a formulé l'hypothèse qu'aux nombres de Reynolds assez élevés les effets visqueux seraient concentrés dans les très petits tourbillons, et que la structure turbulente dans cette partie du spectre atteindrait une condition de « similitude universelle » ou d'équilibre statistique qui ne dépend que de la fourniture cinématique d'énergie $S$ et la viscosité cinématique $\nu$, sur quoi $S=\varepsilon / \rho$. Cette hypothèse a recu une sérieuse confirmation expérimentale. C'est d'un intérêt spécial de noter que la portion du spectre contenant la majeure partie de l'énergie (celle montrée en fig. 11) est bien distincte de celle dans laquelle la dissipation a lieu (qui est réellement bien à droite de la partie montrée dans la figure). On ne peut s'attendre qu'à cette situation, ainsi que cela découle d'une simple comparaison des quantités $E(k)$ et $k^{2} E(k)$ qui caractérisent les deux zones spectrales.

De telles conclusions, au sujet de la turbulence isotrope homogène, auraient peu de signification pour le phénomène anisotrope non homogène dont il s'agit, s'il n'y avait pas deux facteurs influant sur le problème : en premicr lieu, il existe, en général, assez de similitude qualitative entre les deux sortes de turbulence, pour pouvoir faire une interprétation utile; en second lieu, cette similitude semble prendre des aspects quantitatifs lorsque le nombre d'onde devient très grand. Dans un écoulement quelconque, par exemple, il est raisonnable de conclure [21], qu'à tous les nombres de Reynolds, sauf les petits, la structure des grands tourbillons dépend presque entièrement de la forme de l'écoulement moyen lui-même. En outre, plus grand est le nombre de Reynolds, plus petite doit être la taille des tourbillons à laquelle la viscosité commence à jouer un rôle appréciable. Les tensions aux échelles plus grandes que celles-ci sont par conséquent dues à l'inertie, et leur effet est de produire une rupture continue du mouvement tourbillonnaire dans la direction de nombres d'onde plus élevés. Alors, de même que la source des tourbillons dans une zone de rapide variation de vitesse est l'écoulement primaire, on peut s'imaginer que les tourbillons voisins produisent eux-mêmes des zones de tension qui engendrent des tourbillons encore plus petits. Plus grande est la dispersion sur laquelle ce transfert d'énergie a agi, et moins évidentes doivent être les préférences di- 
rectionnelles de la source originaire. En d'autres termes, dans un champ turbulent donné, plus petite est l'échelle de certains tourbillons, plus proche d'un état homogène et isotrope doil être le mouvement dans ces tourbillons. En même temps, l'effet dissipateur de la viscosité devrait croître pendant que l'échelle décroit (en fait presque comme le carré du nombre d'onde), et aux nombres de Reynolds suffisamment grands, la dissipation doit être à peu près toute concentrée dans la portion du spectre dans laquelle la turbulence est essentiellement isotrope.

La conclusion significative à laquelle on est ainsi arrivé est que même dans les zones de turbulence anisotrope non homogène, il peut être possible d'évaluer la dissipation en fonction d'une seule dérivée de vitesse au lieu des neuf qui se trouvent dans la fonction générale de dissipation. Il est déjà évident [31] que cette conclusion est valable pour des cas rclativement simples de turbulence libre avec les tensions de Reynolds, bien que cela ne soit pas nécessairement vrai pour l'écoulement près des parois [32, 33]. Malheureusement, des études même plus poussées que celles-ci ne peuvent être utilisées facilement pour vérifier la théorie dans les conditions plus complexes qu'elles représentent, car des limitations pratiques rendent impossibles à mesurer d'autres termes encore, qu'on ne peut que supposer négligeable. L'hypothèse selon laquelle la théorie est valable est donc essentielle pour conduire l'analyse dans son état actuel d'approximation.

(A suive.)

\section{NOTRE FRONTISPICE}

(Cf. p. 220)

\section{BEI ANGER (1789-1874)}

Tngénieur des Ponts et Chaussées, Bélanger s'adonna à l'hydrau lique surtout pendant les premières années de sa carrière, lorsqu'il fut détaché, en 1823 , à la Compannie concessionnaire du canal dit du duc d'Angonlême, devenu le canal de la Somme, puis, en 1826, à la Compagnie du canal des Ardennes; et c'est de cette période que date son Essai sur la solution numérique dc onclques problcmes relatifs an montement permanent des caux courantes, paru en 1828 et renfermant un procédé de calcul qui, diton à l'époque, "recut l'approbation des géomètres".. Bélanger $y$ exposait en particulier une méthode élémentaire de calcul des courbes de remous, préfigurant les méthodes classiques actuelles et basée sur ure équation qui s'inspirait des travaux de Prony sur l'expression du frottement en écoulement uniforme.

Jean- Japtiste Charles Joseph Bélanger, né à Valenciennes le 4 avril 1789, élève de l'Ecole P'olytechnique, pris de celle de Ponts et Chaussées, débuta en 1816 à La Réole dans le Corps des Ponts et Chaussees, qu'il quitta bientot pour les deux misSions rappelees ci-dessus, tuis pour entrer dans l'enseignement. Longtemps inspectent des etudes à l'Ecole Centrale des Arts et mécanique à l'Ecole des Ponts et Chaussées, et, de 1851 à 1861, ì l'Ecole Polytechnique.

Dans ses conférences à la prepnière de ces deux Ecoles, il intro duisit la notion de débit maximum sur un déversoir à large seuil pour lequel il établit cleux équations.

11 était ingénieur en chef depuis 1843. Outre le mémoire cité plus haut, on lui doit des traités de géométrie analytique (1842) de mécanique (dynamique et statique générale) (1847) et de ciné matique (1867); des mémoires traitant de la Théorie de la résis tance et de la fiexion plane des solides (1858), et De l'équivalent mécanique de la chaleur (1863). Bélanger mourut à Neuilly-surSeine en 1874 .

\section{BELANGIER (1789-1874}

Belanger was most concerned roith hydraulics duing the carly ycars of his career wohen in 1823, he was detached from the Corps des Ponts at Chaussees to twork with the Duc d'Angouleme Canal Company, which became the Somme Canal Company, and then with the Ardennes Canal Company in 1826. His "Essa sur la solution numérique de quelques problèmes relatifs au mouvement permanent des eaux courantes $)$, which dates from this period, was published in 1828 and introduced a method of computation which was described by a contemporary as having "recei" ed the approval of geometers"... In it, Bélanger describes an elementary method of computing backwater curves which zoas a forernnuer of presentday standard methods and rohich is based on an equation that owes much to Prony's work on friction assoriated with uniform flow.

Born at Valenciennes on April 4th 1789. Jean-Baptiste Bélanger studied at the Ecole Polytechrique and then at the Ecole des Ponts et Chaussées. In 1816, before going to work with the canal companies referred to earlicr. he became a member of the Corps des Ponts et Chaussées at La Réole. He subsequently entered the teaching profession. For a long time he was Inspector of Studies at the Ecole Centrale des Arts ct Manufactures until he became Professor of Mechanics at the Ecole des Ponts et Chaussées in 1841. He zeas Professor of Mechanics at the Ecole Polytechuique from 1851 to 1861 .

It woas during his lectures at the first of these colleges that he introduced the idea of a matimum discharge over a broad-crested weir for wich he derived two equations.

He was chief engineer from 1843 onterds. In addition to the paper mentioned earlier, he zorote textbooks on analytical geometry (1842), on dynamics and statics (1847) and on kinematics (1867). Also due to him are papers on "La théorie de la résistance et de la flexion plane des solides 》 (1858), and "De l'équivalent mécanique de la chaleur» (1863). Bélanger died at Neuilly-sur-Seine in 1874 . 


\title{
Distribution of energy in regions of separation
}

\author{
by Hunter ROUSE
}

IOWA INSTITUTE OF HYDRAULIC RESEARCH STATE UNIVERSITY OF JOWA

During the past decade the lowa Institute of Hydraulic Research has undertaken a series of studies of flow in the vicinity of quasi-stable eddies produced by separation at boundary angularities.

Professor Rouse described in the present paper the determination of the mean and secondary patterns of axisymmetric flow for two comparable boundary forms: the abrupt inlet and the blunt shaft Measurements available for analysis included the distributions of mean velocity, mean pressure, longitudinal and radial intensities of turbulence, turbulent shear, and longitudinal intensity gradient. Through use of the equations of momentum and of energy for the mean and the secondary motion, the measured distributions were adjusted to yield the required balance of the essential terms in the equations, thus yielding results in general accord with physical requirements. There are presented in the form of the flow patterns themselves, supplentented by curves of variation of the individual momentum and energy terms throughout the regions of separation.

of particular significance is the interrelationship of turbulence production, convection, and dissipation, and the fact that a relatively small amount of turbulence energy is required locally to produce a large overall change in flow pattern.

Two final diagrams indicate the variation of the terms of the Bernoulli equation extended to include the effects of turbulence and energy dissipation.

For illustrations, see French text, p. 221

\section{INTRODUCTION}

A very common characteristic of flow phenomena in the field of hydraulics is the local separation of the main stream from the boundary. Whether for reasons of economy, disinterest on the part of the designer, or technical impracticability, the streamlining of hydraulic siructures to eliminate this occurrence is still the exception rather than the rule. Plumbing fixtures, channel transitions, conduit inlets, and bridge abutments are thus made square-edged more often than round, with the accompanying vibration, eddy loss, or erosion that separation usually entails. Sometimes, to be sure, separation and eddy formation are necessary features of the flow system, as in the case of valves, baffles, and stilling basins. Moreover, sharpedged boundary deflections are in certain inslances preferable to those with small but finite radii of curvature, since the latter can produce intermittency of the separation process, which has little in its favor and very much against it. In any event, the mechanics of flow in a region of separation would appear in many ways to be more important to the hydraulician than to any other scientist or engineer. This is perhaps the reason for the fact that such flow has in the past received so little theoretical attenlion. Surely, however, it is a reason for the hydraulician to take a prominent part in its further investigation.

Hydraulics has long concerned itself almost exclusively with mean-flow analyses, thereby disregarding the secondary or turbulent motion superposed upon the primary. Of recent years other professions have given considerable attention to fluid turbulence itself, so restricting the phenomenon that the mean flow could be disregarded. Through such simplifications much has been learned both about the mean flows and about the basic nature of turbulence. In most actual states of fluid motion, nevertheless, neither aspect can be studied satisfactorily by itself, for each has a part in determining the characteristics of the other. This is particularly true of the separation phenomenon in its broadest aspects.

Considerable progress has been made in aerodynamics in predicting the point of separation on a gently curved boundary as a function of the boundary geometry and the Reynolds number-provided that the separation as it occurs either does not appreciably affect the surrounding flow or affects it in a manner that can be approximated from free-streamline analysis. Yet for the simple boundary arrangement shown in Fig. 1, in which the point of separation is fixed, not only can the mean shape of the separation zone not be accurately predicted, but even with such knowledge the velocity and pressure distribution within the eddy and the energy loss which its presence entails could be estimated only very roughly; and such secondary-flow characteristics as the intensity of the turbulence within and in the wake of the eddy and the frequency of fuctuations at its downstream end could only be guessed at.

The role of such eddies in energy transformation has long been undervalued by some, yet overvalued by others. Because the mean flow pattern necessarily indicates a stable vortex with as definite a system of streamlines as that of the outside flow, the temptation is to attribute to the eddy the same energy per unit volume as is possessed by the flow itself. This is a definite fallacy, for the line of separation is not a free streamline but one along which the energy stead- 
ily decreases. Yet because the eddy is actually a fluctuating source of smaller eddies shed into its wake, it is often visualized as a sort of vortex mill at all points of which turbulence is produced and energy thereby lost. As a matter of fact, the primary zone of turbulence production lies between the eddy and the main flow, just as in any case of mixing across a surface of velocity discontinuity, and the turbulence is convected and diffused before its energy is fully dissipated. It is the return flow which distinguishes the phenomenon from the simpler cases of mixing: on the one hand, the fluid re-entrained on the eddy side is already highly turbulent; on the other hand, the downstream end of the eddy fluctuates in position as appreciable parts of it intermittently pass off into the main stream.

The problem is evidently well removed from the two states of motion with which some analytical success has already been had. It differs from the isotropic case, typified by normal planes of flow behind a grid, in that it is a problem of pronounced anisotropic or shear flow; and it differs from the similarity case, typified by established flow in a wake or pipe, because it is specifically a localized occurrence, however far-reaching its effect downstream may be. For want of ready analytical tools, the investigation of the phenomenon must proceed in a quasi-empirical way: one must examine pertinent cases in as great experimental detail as possible; estimate as yet unmeasurable characteristics through correlation of measurements with the equations of motion; and seek, as the mechanism of the eddy motion becomes familiar, to accomplish its expression in approximate mathematical form.

Under the writer's direction several exploratory studies of this sort have already been con- ducted in the laboratories of the Iowa Institute of Hydraulic Research. The first was a doctoral project carried out by H.C. Hsu [1] involving: the separation at an abrupt two-dimensional conduit expansion; the characteristics of the mean flow and of the turbulence were measured and correlated by means of the equations of motion, so that the transformation of the energy from the primary through the secondary motion to its ultimate thermal dissipation could be represented in gross detail. This was followed by an investigation by M. Arie [2] of the flow behind a normal wall [3], the results also being analyzed in energy form [4]. Similar studies, though less dependent upon boundary geometry, were those by $O$. Erickson and the writer of a normal jet in a cross flow [5], by T.T. Siao [6] and S. Nagaratnam [7] of the hydraulic jump [8], and by T.K. Rao [9] of a jet directed axially into a counterflow. Each of these cases had the common attribute of a stable zone of backflow a standing eddy - along the edge of which turbulence was generated, the subsequent convection, diffusion, and decay of which were essential factors in the determination of the entire flow pattern.

As a further step toward the ultimate understanding of this phenomenon, the present paper describes an investigation of axisymmetric flow at two comparable boundary configurations of basic interest in hydraulics and related fields : a pipe inlet and a blunt-nosed shaft. Both the mean and the secondary patterns of flow are explored, and an effort is made to trace the course of energy transfer from the mean flow through the turbulence to its final form of heat. With these flows as specific examples, the significance of the energy relationships is discussed in considerable detail, to the end of facilitating additional studies of this nature.

\section{PRELIMINARY EXAMINATION OF THE FLOWS TO BE INVESTIGATED}

An abrupt inlet to a pipe in the wall of a large reservoir and a cylindrical shaft with blunt upstream end are boundary forms that seem at first glance to be far removed from one another, for the one is characteristic of passages through which fluid moves in a confined state and the other of bodies which more generally move through fluids of great extent. The principle of relative motion, of course, easily eliminates the one apparent distinction. As for the other, however, not until such axisymmetric boundary forms are examined in terms of their two-dimensional counterparts does their actual similarity become evident. In fact, such representation serves a double purpose, for it is only the two-dimensional counterparts that are even approximately amenable to mathematical analysis, and this approximation is desirable for at least a qualitative understanding of the physical principles involved in the respective flows.

With reference to Fig. 2, it will be seen that the repeating elements of flow past an array of sharp-edged normal plates can be regarded in either of two ways. Thus the element ABCD can be combined with its mirror image above to form a passage symmetrical about the line $A B$ representative of confined flow around a plate, the line CD and its image thereby defining solid boundaries. Similarly, CD may be taken as the line of symmetry, solid boundaries along 
line $A B$ and its image, logether with the two half plates, simulating a passage with a local constriction on each wall. If one disregard for the moment the interaction of the neighboring eddy zones downstream, the lines of separation would appear to be the same in either case. The true distinction between the two points of view becomes evident only as the plate half-width $a$ and half-spacing $b$ are made increasingly unequal. As the ratio $a / b$ becomes much less than unity, for example, the immersed-body aspect of the array is accentuated; and as the ratio becomes very great, it is the efflux aspect that is paramount. The limit $a / b=0$ of the first trend, of course, is the plate of finite width in an infinite fluid, whereas the limit $a / b=\infty$ of the other trend is the slot of finite width in an infinite wall.

By the method of conformal analysis, coupled with the free-streamline assumption, von Mises [10] was able to evaluate the contraction ratio $b_{b} / b$ of the free jet for any value of the boundary ratio $a / b$, including of course the Kirchhoff limit $\pi /(\pi+2)=0.61$ for $a / b=\infty$. His results have been closely approximated by experiments with both free and submerged jets. Application of the same method of analysis to flow around a plate, however, yields results that are verified only for a liquid-gas interface (i.e. a cavitation pocket) of very great extent, since only then does the pressure at the interface approach that of the ambient flow. As has been shown by Riabouchinsky [11], on the other hand, the assumplion of either a lower pressure or a more rapid curvature of the interfacial profile will permit conformal methods to yield results more nearly in accord with experiment.

Such accord is, to be sure, merely a gross one, for it deals with overall forces or changes of pressure rather than local details of the flow pattern. The latter, in fact, are quite contrary to the free-streamline assumption except in the immediate neighborhood of the separation point. Because of the high shear along the unguided surface of separation, except at low Reynolds numbers this is the seat of intense generation of lurbulence, which diffuses laterally as it is carried downstream and thereby changes the details of an ever-widening zone. In the limiting cases $a / b=0$ and $a / b=\infty$, the patterns are those of the expanding wake and the expanding jet, respectively, the approximate solutions of which [12] have been known for several decades. The basis of such solutions is the experimentally observed similarity of the velocity-distribution functions at successive cross sections well downstream from the initial non-uniformities. In their immediate vicinity, of course, conditions of similarity do not yet exist in either instance.

Much the same considerations apply to flow past the cascade of hars shown in Fig. 3, for in the one limit it can be assumed to represent flow from a reservoir into a parallel conduit and in the other limit unconfined flow past an isolated pier or wall. These too lead ultimately lo states of motion that have been analyzed with success in terms of similarity distributions, uniform two-dimensional flow being the one limit and boundary-layer flow along a flat plate the other. In the immediate vicinity of the initial sections, however, neither does similarity exist nor are conformal methods of much use in more than a qualitative sense. On the other hand, it is evident that the two states of motion have much in common, for a finite length of separation pocket must necesssarily exist in each, and occurrences between the boundary and the edge of the pocket must be essentially the same. In fact, it is outside the zone of separation that the basic distinction is to be found, for beyond the one lies a great expanse of relatively undisturbed fluid, whereas just across from the other is the same zone of separation in mirror image.

If the axisymmetric counterparts of these patterns (see Figs. 4 and 5) are now considered, it will be seen that the pertinent similarities of the two-dimensional cases are all retained, while the dissimilarities are further accentuated. Evidently, the axisymmetric cases can no longer be regarded as the limits of a parametric Irend; on the contrary, each is in effect the "insideout" version of the other. Though these cases are even less subject to rigorous analysis than their two-dimensional counterparts, the same general observations apply, and it will be seen that still others can be brought to bear.

If one disregard the effect of viscous shear along the wall and the ever-increasing deviation of the streamlines from a purely linear course, flow at the rate $Q$ toward a circular outlet in the wall of a reservoir can be defined through use of the stream function

$$
\psi=\frac{Q}{2 \pi} \frac{x}{\sqrt{x^{2}+r^{2}}}
$$

for the point sink, for which the velocities arc, as usual, $u=\partial \psi / r \partial r$ in the axial direction $x$ and $v=-\partial \psi / r \partial x$ in the radial direction $r$. Since the flow is rapidly accelerated, the effect of viscous shear can in fact be ignored without appreciable error, but the deviation of the streamlines becomes rather important as the inlet is approached. This discrepancy could be partially eliminated through use of the stream function for a distribution of sinks over the plane of the opening; however, experimental knowledge of the flow pattern (or at least of the surface of separation) is necessary to determine the required distribu- 
tion function, and the matter is hence of academic rather than practical interest. On the other hand, it is known from numerical analyses [13] that the contraction coefficients for circular orifices, taken as areal ratios, are very nearly if not exactly the same as those for slots having the same linear proportions; at the limit, for example, $\left(d_{c} / d\right)^{2}=0.61$. Moreover, experiments [14] have shown that this value is nearly as correct for the submerged jet as for the free. On the assumption that the same degree of contraction is evidenced by the flow at a pipe inlet, for almost a century the resulting Borda loss

$$
\Delta H=\frac{\left(U_{1}-U_{2}\right)^{2}}{2 g}
$$

has been combined with the limiting contraction ratio

$$
\left(\frac{d_{c}}{d}\right)^{2}=0.61=\frac{U}{U_{c}} \approx \frac{U_{2}}{U_{1}}
$$

to yield for the head lost at a pipe inlet the value

$$
\frac{\Delta H}{U^{2} / 2 g}=1-0.61 \approx 0.5
$$

which is that customarily used in hydraulic design. Except for a few measurements of the pressure distribution in a short tube, indicated in Fig. 4 [15], and innumerable measurements of both pressure and velocity far downstream from an inlet [16], little further seems to be known of the flow under consideration.

Even less is known about flow past a blunl shaft, at least from the historical point of view, for only in connection with projectile cavitation has it been investigated, and that only within the last decade or so. To proceed with the discussion in the same manner, however, it may be noted [17] that experiments have shown the separation zone behind a disk to have a diameter perhaps 1.7 times that of the disk, the drag coefficient to have the value

$$
\frac{F /\left(\pi d^{2} / 4\right)}{\rho U^{2} / 2}=1.20
$$

and the pressure coefficient for the immediate wake to be

$$
\frac{p}{U^{2} / 2}=-0.45
$$

Measurements of the pressure distribution on the blunt shaft [18] are as sketched in Fig. 5, the pocket diameter being $1.5 d$ and the minimum pressure ratio being- 0.62 . If shear on the surface of the cylinder is ignored, the drag coefficient of the shaft should be only 0.78 , resulting from the pressure distribution on the front face alone. For those sections of the wake over which the pressure decrement can be ignored, this should correspond to the momentum integral [19]:

$$
F=2 \pi \rho \int_{d / 2}^{\infty} u(U-u) r d r
$$

Were the form of the separation zone and of the subsequent velocity distribution known even approximately, so that the corresponding displacement thickness of the disturbance could be computed, the pattern of the surrounding irrotational flow (except perhaps for the immediate vicinity of the blunt face) could be expressed in terms of the stream function for a distribution of doublets $[3,19]$ of unit strengh $\Delta(t)$ from $x=t=a$ to $b$ along the line of symmetry :

$$
\psi=r^{2} U\left\{1 / 2-\int_{a}^{b} \frac{\Delta(t)}{\left[(t-x)^{2}+r^{2}\right]^{3 / 2}} d t\right\}
$$

Obviously, this would again require a considerable amount of experimental information, though the limiting part of it is at hand for the boundary layer on a cylindrical shaft [20].

The two cases of flow under study are evidently subject to mathematical description in the irrotationnel regions upstream, provided that certain key data are given by experiment; and considerable knowledge of an analytical as well as experimental nature is already available for the rolational zones of similarity well downstream. To date, however, only a qualitative picture of the transition between the initial and final parts of the patterns can be obtained without detailed measurement. The phenomena of turbulence production and dissipation are known to be extremely complex, moreover, and unless one turn for guidance to at least the basic hydrodynamic equations, such measurement would certainly be a haphazard process. For this reason considerable attention will next be given to the implications of these equations.

\section{FORMULATION AND INTERPRETATION OF THE ENERGY RELATIONSHIPS}

\section{Equations of Stokes and Reynolds}

The basic equations of motion in terms of the cylindrical coordinates $x, r$, and $\theta$ are derived by equating to the total rates of change of the corresponding velocity components $u, v$, and $w$ the 
respective components of the force per unit mass acting upon a fluid element such as that shown in Fig. 6. As the volume of the element approaches the limit zero, these equations reduce to the form [21]:

$$
\left.\begin{array}{l}
\frac{\partial u}{\partial t}+u \frac{\partial u}{\partial x}+v \frac{\partial u}{\partial r}+w \frac{\partial u}{r \partial \theta}=-\frac{\partial \Omega}{\partial x}+\frac{1}{r}\left[\frac{\partial\left(r \sigma_{x}\right)}{\partial x}+\frac{\partial\left(r \tau_{r, x}\right)}{\partial r}+\frac{\partial\left(r \tau_{\theta r}\right)}{r \partial \theta}\right] \\
\frac{\partial v}{\partial t}+u \frac{\partial v}{\partial x}+v \frac{\partial v}{\partial r}+w \frac{\partial v}{r \partial \theta}-\frac{w^{2}}{r}=-\frac{\partial \Omega}{\partial r}+\frac{1}{r}\left[\frac{\partial\left(r \tau_{x r}\right)}{\partial x}+\frac{\partial\left(r \sigma_{r}\right)}{\partial r}+\frac{\partial\left(r \tau_{\theta r}\right)}{r \partial \theta}-\sigma_{\theta}\right] \\
\frac{\partial w}{\partial t}+u \frac{\partial w}{\partial x}+v \frac{\partial w}{\partial r}+w \frac{\partial w}{r \partial \theta}+\frac{v w}{r}=-\frac{\partial \Omega}{r \partial \theta}+\frac{1}{r}\left[\frac{\partial\left(r \tau_{x \theta}\right)}{\partial x}+\frac{\partial\left(r \tau_{r \theta}\right)}{\partial r}+\frac{\partial\left(r \sigma_{\theta}\right)}{r \partial \theta}+\tau_{r \theta}\right]
\end{array}\right\}
$$

Herein $\Omega$ represents the gravitational potential (elevation times effective fluid weight per unit mass); $\sigma_{v}$ is the normal (tensile) stress per unit area on a surface normal to the $x$-axis, necessarily in the $x$-direction; $\tau_{r x}$ is the tangential stress per unit area on a surface normal to the $r$-axis in the $x$-direction; and so on. For reasons of equilibrium, $\tau_{r x}=\tau_{x r}$, etc. To the equations of motion should be added the equation of continuity,

$$
\frac{\partial(r u)}{\partial x}+\frac{\partial(r v)}{\partial r}+\frac{\partial(r w)}{r \partial \theta}=0
$$

which is derived by expressing either the fact that the volume of a fluid element of constant density $p$ must remain unchanged during movement from point to point, or its equivalent that the instantaneous rate of flow out of an elementary region of space must equal simultaneously the rate of flow into it.

Approximately a century age Stokes [22] expressed the foregoing intensities of stress in terms of the dynamic viscosity $\mu$ as follows :

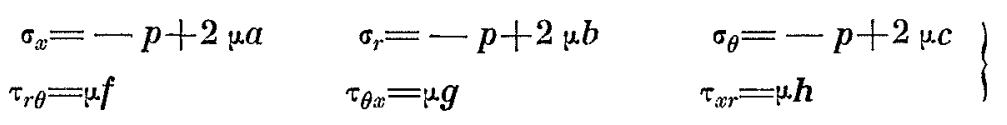

Herein $p$ represents the mean intensity of pressure,

$$
p=-\frac{\sigma_{x}+\sigma_{r}+\sigma_{\theta}}{3}
$$

and the letters $a-h$ denote the rates of linear and angular deformation :

$$
\left.\begin{array}{llr}
a=\frac{\partial u}{\partial x} & b=\frac{\partial v}{\partial r} & c=\frac{\partial w}{r \partial \theta}+\frac{v}{r} \\
i=\frac{\partial v}{r \partial \theta}+\frac{\partial w}{\partial r}-\frac{w}{r} & g=\frac{\partial w}{\partial x}+\frac{\partial u}{r \partial \theta} & h=\frac{\partial u}{\partial r}+\frac{\partial v}{\partial x}
\end{array}\right\}
$$

Introduction of these stresses into the basic equations of motion resulted in what have come to be called the equations of Navier-Stokes :

$$
\begin{gathered}
\rho\left(\frac{\partial u}{\partial t}+u \frac{\partial u}{\partial x}+v \frac{\partial u}{\partial r}+w \frac{\partial u}{r \partial \theta}\right)=-\frac{\partial}{\partial x}(p+\rho \Omega)+\mu\left(\frac{\partial^{2} u}{\partial x^{2}}+\frac{\partial^{2} u}{\partial r^{2}}+\frac{1}{r} \frac{\partial u}{\partial r}+\frac{\partial^{2} u}{r^{2} \partial \theta^{2}}\right) \\
P\left(\frac{\partial v}{\partial t}+u \frac{\partial v}{\partial x}+v \frac{\partial v}{\partial r}+w \frac{\partial v}{r \partial \theta}-\frac{w^{2}}{r}\right) \\
=-\frac{\partial}{\partial r}(p+\rho \Omega)+\mu\left(\frac{\partial^{2} v}{\partial x^{2}}+\frac{\partial^{2} v}{\partial r^{2}}+\frac{1}{r} \frac{\partial v}{\partial r}-\frac{v}{r^{2}}+\frac{\partial^{2} v}{r^{2} \partial \theta^{2}}-\frac{2}{r} \frac{\partial w}{r \partial \theta}\right) \\
\rho\left(\frac{\partial w}{\partial t}+u \frac{\partial w}{\partial x}+v \frac{\partial w}{\partial r}+w \frac{\partial w}{r \partial \theta}+\frac{v w}{r}\right) \\
=-\frac{\partial}{r \partial \theta}(p+\rho \Omega)+\mu\left(\frac{\partial^{2} w}{\partial x^{2}}+\frac{\partial^{2} w}{\partial r^{2}}+\frac{1}{r} \frac{\partial w}{\partial r}-\frac{w}{r^{2}}+\frac{\partial^{2} w}{r^{2} \partial \theta^{2}}+\frac{2}{r} \frac{\partial v}{r \partial v}\right)
\end{gathered}
$$

Were each of these equations to be multiplied by the corresponding velocity component and the three then added together, the left side of the sum would be found to represent, per unit volume, 
the rate at which the kinetic energy of the fluid is changing, and the right side the corresponding rate at which work is being done. The relationship is thus a conservative one. The total rate at which work is being done within the fluid, on the other hand, is found by multiplying the stress on each face of a typical element by the corresponding velocity component, adding the products algebraically, and dividing by the volume of the element. If the conservative rate of doing work is now subtracted from the total, the result will represent the rate at which energy is being dissipated by viscous action [23] :

$$
\begin{aligned}
& \Phi=\mu\left(2 a^{2}+2 b^{2}+2 c^{2}+f^{2}+g^{2}+h^{2}\right) \\
& =i^{\mu}\left[\left(\frac{\partial u}{\partial x}\right)^{2}+\left(\frac{\partial v}{\partial x}\right)^{2}+\left(\frac{\partial w}{\partial x}\right)^{2}+\left(\frac{\partial u}{\partial r}\right)^{2}+\left(\frac{\partial v}{\partial r}\right)^{2}+\left(\frac{\partial w}{\partial r}\right)^{2}+\left(\frac{\partial u}{r \partial \theta}\right)^{2}+\left(\frac{\partial v}{r \partial \theta}\right)^{2}+\left(\frac{\partial w}{r \partial \theta}\right)^{2}\right]
\end{aligned}
$$

Reynolds [24] adapted the equations of Stokes to the study of turbulent flow by introducing a mean value and an instantaneous deviation from the mean for the pressure intensity and each of the three velocity components, and by subsequently eliminating all terms of which the temporal mean value was necessarily zero. If the mean values are indicated as usual by bar's and the devialions by primes, and if, for further simplicity, the equations are reduced to those pertinent to problems of steady mean flow with a zero mean velocity in the $\theta$-direction, the Reynolds equations will be :

$$
\begin{aligned}
& \rho\left(i \bar{u} \frac{\partial \bar{u}}{\partial x}+\bar{v} \frac{\partial u}{\partial r}+\overline{u^{\prime} \frac{\partial u^{\prime}}{\partial x}}+\overline{v^{\prime} \frac{\partial u^{\prime}}{\partial r}}+\overline{w^{\prime} \frac{\partial u^{\prime}}{r \partial \theta}}\right)=-\frac{\partial}{\partial x}(\bar{p}+\rho \Omega)+\mu\left(\frac{\partial^{2} \bar{u}}{\partial x^{2}}+\frac{\partial^{2} \bar{u}}{\partial r^{2}}+\frac{1}{r} \frac{\partial u}{\partial r}\right) \\
& \rho\left(\bar{u} \frac{\partial \bar{u}}{\partial x}+\bar{v} \frac{\partial \ddot{v}}{\partial r}+\overline{u^{\prime} \frac{\partial v^{\prime}}{\partial x}}+\overline{v^{\prime} \frac{\partial v^{\prime}}{\partial r^{r}}}+\overline{u^{\prime} \frac{\partial v^{\prime}}{r^{\prime} \partial \theta}-\overline{w^{\prime 2}}}\right)=-\frac{\partial}{\partial r}(\bar{p}+\rho \Omega)+\mu\left(\frac{\partial^{2} \bar{v}}{\partial x^{2}}+\frac{\partial^{2} \bar{v}}{\partial r^{2}}+\frac{1}{r} \frac{\partial \bar{v}}{\partial r}-\frac{\bar{u}}{r^{2}}\right)
\end{aligned}
$$

The corresponding equation of continuity is then simply :

$$
\frac{\partial(r \bar{u})}{\partial x}+\frac{\partial(r \bar{v})}{\partial r}=0
$$

At first glance the equations appear to have changed only through the replacement of instantaneous values by mean values and through the addition of several terms involving mean combinations of the fluctuations. The right sides of the equations have thus become simplified by the elimination of the fluctuating viscous stresses, which-however complicated they may be- necessarily have a temporal mean value of zero. However, fluctuating accelerative terms, which do not necessarily have a mean value of zero, have made the left sides that much more conplicated. As a matter of fact, these accelerative terms can be construed as mean rates of momentum transfer, and hence (by analogy to the theory of molecular viscosity) as stresses due to mixing on a molar rather than a molecular scale. This is clarified by the following manipulation of the quantities in question:

$$
\left.\begin{array}{r}
u^{\prime} \frac{\partial u^{\prime}}{\partial x}+v^{\prime} \frac{\partial u^{\prime}}{\partial r}+w^{\prime} \frac{\partial u^{\prime}}{r \partial \theta}=\frac{\partial\left(u^{\prime} u^{\prime}\right)}{\partial x}+\frac{\partial\left(u^{\prime} v^{\prime}\right)}{\partial r}+\frac{\partial\left(u^{\prime} w^{\prime}\right)}{r \partial \theta}-u^{\prime}\left(\frac{\partial u^{\prime}}{\partial x}+\frac{\partial v^{\prime}}{\partial r}+\frac{v^{\prime}}{r}+\frac{\partial w^{\prime}}{r \partial \theta}\right)+u^{\prime} \frac{v^{\prime}}{r} \\
\boldsymbol{u}^{\prime} \frac{\partial v^{\prime}}{\partial x}+v^{\prime} \frac{\partial v^{\prime}}{\partial r}+w^{\prime} \frac{\partial v^{\prime}}{r \partial \theta}-\frac{w^{\prime 2}}{r}=\frac{\partial\left(u^{\prime} v^{\prime}\right)}{\partial x}+\frac{\partial\left(v^{\prime} v^{\prime}\right)}{\partial r}+\frac{\partial\left(v^{\prime} w^{\prime}\right)}{r \partial \theta} \\
-v^{\prime}\left(\frac{\partial u^{\prime}}{\partial x}+\frac{\partial v^{\prime}}{\partial r}+\frac{v^{\prime}}{r}+\frac{\partial w^{\prime}}{r \partial \theta}\right)+v^{\prime} \frac{v^{\prime}}{r}+\frac{w^{\prime} w^{\prime}}{r}
\end{array}\right\}
$$

The lerms within the large parentheses are zero by virtue of the continuity equation, and the products of $\rho$ and the terms remaining-logically called the Reynolds stresses-will all be found analogous to the Stokes stresses written in terms of the mean velocily. In other words

$$
\left.\begin{array}{c}
\overline{\sigma_{r}}=-p+2 \mu \frac{\partial \bar{u}}{\partial x}-\bar{q} \overline{u^{2}} ; \quad \bar{\tau}=-p+2 \mu \frac{\partial \bar{v}}{\partial r}-\rho \overline{v^{2}} ; \quad \bar{\sigma}_{\theta}=-p+2 \mu \frac{\bar{i}}{r}-\overline{\rho \boldsymbol{w}^{\prime 2}} \\
\bar{\tau}_{r \theta}=0 ; \quad \bar{\tau}_{\theta x}=0 ; \quad \bar{\tau}_{x r}=\bar{\tau}_{r x}=\bar{\tau}=\mu\left(\frac{\partial \bar{u}}{\partial r}+\frac{\partial \bar{v}}{\partial x}\right)-\rho \overline{u^{\prime} v^{\prime}}
\end{array}\right\}
$$

Introduction of these stresses into the Reynolds equations, together with a comparable manipulation of the mean terms on the left, leads to the rather symmetrical form : 


$$
\left.\begin{array}{l}
\frac{\rho}{r}\left[\frac{\partial\left(r u^{2}\right)}{\partial x}+\frac{\partial(r \ddot{u} \bar{v})}{\partial r}\right]=-\rho \frac{\partial \Omega}{\partial x}+\frac{1}{r}\left[\frac{\partial\left(r \overline{\sigma_{x}}\right)}{\partial x}+\frac{\partial(r \tau)}{\partial r}\right] \\
\frac{\rho}{r}\left[\frac{\partial(\dot{r} \tilde{u} \bar{v})}{\partial x}+\frac{\partial\left(r \bar{v}^{2}\right)}{\partial r}\right]=-\rho \frac{\partial \Omega}{\partial r}+\frac{1}{r}\left[\frac{\partial(r \bar{\tau})}{\partial x}+\frac{\partial\left(\bar{r}_{r}\right)}{\partial r}-\bar{\sigma}_{\theta}\right]
\end{array}\right\}
$$

Such equations of acceleration can also be construed as impulse-momentum relationships if the left sides are taken to represent a change in momentum per unit time per unit volume, and the right sides the corresponding impulse per unit time and volume. Any one of them can thus be integrated over a given region of space to produce a momentum relationship of the type frequently used in hydraulics. The volume integral of the Reynolds equation for the axial direction will obviously be :

$$
\int_{F} \frac{1}{r}\left[\frac{\partial\left(r \bar{u}^{2}\right)}{\partial x}+\frac{\partial(r u \bar{v})}{\partial r}\right] d \Psi=-\rho \int_{F} \frac{\partial \Omega}{\partial x} d F+\int_{Y} \frac{1}{r}\left[\frac{\partial\left(\overline{r \sigma_{x}}\right)}{\partial x}+\frac{\partial(\bar{r} \bar{\tau})}{\partial r}\right] d \psi
$$

Through application of the Gaussian relationship

$$
\int_{v} \frac{1}{r}\left[\frac{\partial(r P)}{\partial x}+\frac{\partial(r Q)}{\partial r}\right] d \Psi=\int_{S}\left(P \frac{\partial x}{\partial n}+Q \frac{\partial r}{\partial n}\right) d S
$$

in which $n$ denotes distance normally outward from the surface, the appropriate volume integrals can be changed to surface integrals, with the significant result that :

$$
\int_{S^{\prime}}\left(\bar{u}^{2} \frac{\partial x}{\partial n}+\bar{u} \bar{v} \frac{\partial r}{\partial n}\right) d S=-\int_{\bar{v}} \frac{\partial \Omega}{\partial x} d t+\int_{S}\left(\bar{\sigma}_{\psi^{\prime}} \frac{\partial x}{\partial n}+\bar{\tau} \frac{\partial r}{\partial n}\right) d S
$$

The left side is now seen to represent the change in the flux of the $x$-component of momentum per unit volume as the fluid passes through the region under consideration - i.e., the difference between the rates of momentum efflux and influx. In general, fluid enters or leaves the region at all points of the bounding surface; in hydraulics, however the region is usually limited by solid boundaries on the sides, through which no flow occurs, and by normal sections at the ends, for which only the one velocity component is relevant. The gravitational term is simply the pertinent component of the weight of fluid contained within the region. All internal stresses are evidently ineffective (i. e. counterbalanced). Of the remaining stress terms, the mean viscous part is generally restricted to shear along such boundaries as are in contact with the flow, where the turbulent stresses necessarily disappear; similarly, the mean turbulent part is generally restricted to the normal flow sections, where the mean viscous stresses are negligible in comparison.

\section{Equations of Energy}

Since relationships of the acceleration or momentum type are necessarily written in terms of vecior components, they can involve no direct measure of energy loss, which is a scalar quantity. As in the case of the viscous-flow equations, the energy type of expression for turbulent flow [25] must be obtained by multiplying each equation by the corresponding velocity component (written throughout as the sum of mean and fluctuating parts) and adding the results. (In order to retain all pertinent terms, it must be noted, the multiplication should be performed before temporal averages are taken and any terms are dropped.) The final mean outcome is the total rate of energy change per unit volume :

$$
\begin{aligned}
& \frac{\rho}{2}\left(\bar{u} \frac{\partial \bar{V} 2}{\partial x}+\bar{v} \frac{\partial \bar{V}^{2}}{\partial r}+\bar{u} \frac{\partial \overline{V^{\prime 2}}}{\partial x}+\bar{v} \frac{\partial \overline{V^{\prime 2}}}{\partial r}+\overline{u^{\prime}} \frac{\partial \overline{V^{\prime 2}}}{\partial x}+\overline{v^{\prime}} \frac{\partial \bar{V}^{\prime 2}}{\partial r}+\overline{w^{\prime}} \frac{\overline{\partial V^{\prime 2}}}{r \partial \theta}\right) \\
& =-\bar{u} \frac{\partial}{\partial x}(\bar{p}+\rho \Omega)-\bar{v} \frac{\partial}{\partial r}(\bar{p}+\rho \Omega)-\overline{u^{\prime} \frac{\partial p^{\prime}}{\partial x}}-\overline{v^{\prime} \frac{\partial p^{\prime}}{\partial r}}-\overline{w^{\prime} \frac{\partial p^{\prime}}{r \partial \theta}} \\
& +\mu\left(\bar{u} \frac{\partial^{2} \bar{u}}{\partial x^{2}}+\bar{u} \frac{\partial^{2} \bar{u}}{\partial r^{2}}+\frac{\bar{u}}{r} \frac{\partial \bar{u}}{\partial r}+\bar{v} \frac{\partial^{2} \bar{v}}{\partial x^{2}}+\bar{v} \frac{\partial^{2} \bar{v}}{\partial r^{2}}+\frac{\bar{v}}{r} \frac{\partial \bar{v}}{\partial r}-\frac{\bar{v}^{2}}{r^{2}}\right. \\
& +\overline{u^{\prime} \frac{\partial^{2} u^{\prime}}{\partial x^{2}}}+\overline{u^{\prime} \frac{\partial^{2} u^{\prime}}{\partial r^{2}}}+\overline{\frac{u^{\prime}}{r} \frac{\partial u^{\prime}}{\partial r}}+\overline{u^{\prime} \frac{\partial^{2} u^{\prime}}{r^{2} \partial \theta^{2}}}
\end{aligned}
$$




$$
\begin{aligned}
& +\overline{v^{\prime} \frac{\partial^{2} v^{\prime}}{\partial x^{2}}}+\overline{\overline{v^{\prime}} \frac{\partial^{2} v^{\prime}}{\partial r^{2}}}+\overline{\overline{v^{\prime}} \frac{\partial v^{\prime}}{\partial r}}+\overline{v^{\prime} \frac{\partial^{2} v^{\prime}}{r^{2} \partial \theta^{2}}}-\overline{2 v^{\prime} \frac{\partial w^{\prime}}{r \partial \theta}}-\overline{\frac{v^{\prime 2}}{r^{2}}}
\end{aligned}
$$

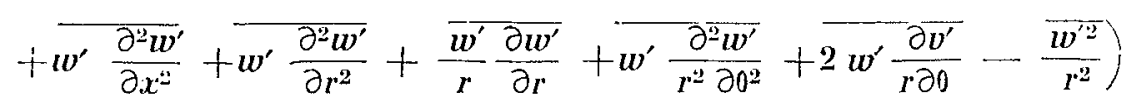

$$
\begin{aligned}
& -e\left(\bar{u} \frac{\partial \overline{u^{\prime 2}}}{\partial x}+\bar{u} \frac{\partial \overline{u^{\prime} v^{\prime}}}{\partial r}+\bar{u} \frac{\overline{u^{\prime} v^{\prime}}}{r}+\bar{v} \frac{\partial \overline{u^{\prime} v^{\prime}}}{\partial x}+\bar{v} \frac{\partial \overline{v^{\prime 2}}}{\partial r}+\bar{v} \frac{\overline{v^{\prime 2}}}{r^{\prime}}\right. \\
& \left.+\overline{u^{\prime 2}} \frac{\partial \bar{u}}{\partial x}+\overline{u^{\prime} v^{\prime}} \frac{\partial \bar{u}}{\partial r}+\overline{u^{\prime} v^{\prime}} \frac{\partial \bar{v}}{\partial x}+\overline{v^{\prime 2}} \frac{\partial \bar{v}}{\partial r}\right)
\end{aligned}
$$

Had the Reynolds equations been multiplied by the corresponding components of the mean velocity and then added, rearrangement of terms would have yielded the following form of the energy equation for the mean motion alone :

$$
\begin{aligned}
& -\rho \bar{u} \frac{\partial \Omega}{\partial x}-\rho \bar{v} \frac{\partial \Omega}{\partial x}+\frac{1}{r}\left[\frac{\partial}{\partial x} r\left(\bar{u} \bar{\sigma}_{x}+\bar{v} \bar{\tau}\right)+\frac{\partial}{\partial r} r\left(\bar{u}+\overline{v \sigma_{r}}\right)\right] \\
& =\frac{\rho}{2}\left(\bar{u} \frac{\partial \bar{V}^{2}}{\partial x}+\bar{v} \frac{\partial \bar{V}^{2}}{\partial r}\right)+\bar{\sigma}_{x} \frac{\partial \bar{u}}{\partial x}+\bar{\sigma}_{r} \frac{\partial \bar{v}}{\partial r}+\bar{\sigma}_{\theta} \frac{\bar{v}}{r}+\bar{\tau}\left(\frac{\partial \bar{u}}{\partial r}+\frac{\partial \bar{v}}{\partial x}\right)
\end{aligned}
$$

In terms of the individual stresses this becomes

$$
\begin{aligned}
& -u \frac{\partial}{\partial x}(\bar{p}+\rho \Omega)-\bar{v} \frac{\partial}{\partial r}(\bar{p}+\odot \Omega) \\
& +\frac{\mu}{r}\left\{\frac{\partial}{\partial x} r\left[2 \bar{u} \frac{\partial \bar{u}}{\partial x}+\bar{v}\left(\frac{\partial \bar{u}}{\partial r}+\frac{\partial \bar{v}}{\partial x}\right)\right]+\frac{\partial}{\partial r} r\left[\bar{u}\left(\frac{\partial \bar{u}}{\partial r}+\frac{\partial \bar{v}}{\partial x}\right)+2 \bar{v} \frac{\partial \bar{v}}{\partial r}\right]\right\} \\
& -\frac{\rho}{r}\left[\frac{\partial}{\partial x} r\left(\bar{u} \overline{u^{\prime 2}}+\bar{v} \overline{u^{\prime} v^{\prime}}\right)+\frac{\partial}{\partial r} r\left(\bar{u} \overline{u^{\prime} v^{\prime}}+\bar{v} \overline{v^{\prime 2}}\right)\right] \\
& =\frac{\rho}{2}\left(\bar{u} \frac{\partial \bar{V}^{2}}{\partial x}+\bar{v} \frac{\partial \bar{v}^{2}}{\partial r}\right)+\mu\left[2\left(\frac{\partial \bar{u}}{\partial x}\right)^{2}+2\left(\frac{\partial \bar{v}}{\partial r}\right)^{2}+2\left(\frac{\bar{v}}{r}\right)^{2}+\left(\frac{\partial \bar{u}}{\partial r}+\frac{\partial \bar{v}}{\partial x}\right)^{2}\right] \\
& -\rho\left[\overline{u^{\prime 2}} \frac{\partial \bar{u}}{\partial x}+\overline{v^{\prime 2}} \frac{\partial \bar{v}}{\partial r}+\overline{w^{\prime 2}} \frac{\bar{v}}{r}+\overline{u^{\prime} v^{\prime}}\left(\frac{\partial \bar{u}}{\partial r}+\frac{\partial \bar{v}}{\partial x}\right)\right]
\end{aligned}
$$

subtraction of which from the equation for the total change yields the equation of energy for the turbulent motion alone :

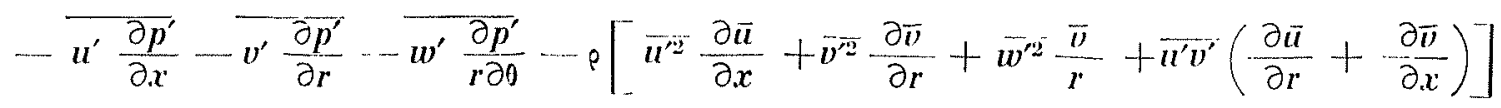

$$
\begin{aligned}
& -\frac{\mu}{r}\left\{\frac{\partial}{\partial x} r\left|\overline{u^{\prime}} \frac{\partial u^{\prime}}{\partial x}+\overline{-v^{\prime}\left(\frac{\partial u^{\prime}}{\partial r}+\frac{\partial v^{\prime}}{\partial x}\right)}+\overline{w^{\prime}\left(\frac{\partial w^{\prime}}{\partial x}+\frac{\partial u^{\prime}}{r \partial g}\right)}\right|\right. \\
& +\frac{\partial}{\partial r} r\left[\overline{u^{\prime}\left(\frac{\partial u^{\prime}}{\partial r}+\frac{\partial v^{\prime}}{\partial x}\right)}+\overline{v^{\prime} \frac{\partial v^{\prime}}{\partial r}}+\overline{w^{\prime}\left(\frac{\partial v^{\prime}}{r \partial \theta}+\frac{\partial w^{\prime}}{\partial r}-\frac{w^{\prime}}{r}\right)}\right] \\
& \left.+\frac{\partial}{r \partial \theta} r\left[\overline{u^{\prime}\left(\frac{\partial w^{\prime}}{\partial x}+\frac{\partial u^{\prime}}{r \partial \theta}\right)} \overline{+v^{\prime}\left(\frac{\partial v^{\prime}}{r \partial \theta}+\frac{\partial w^{\prime}}{\partial r}-\frac{w^{\prime}}{r}\right)}+\overline{w^{\prime}\left(\frac{\partial w^{\prime}}{r \partial \theta}+\frac{v^{\prime}}{r}\right)}\right]\right\} \\
& =\frac{\rho}{2}\left(\bar{u} \frac{\partial \overline{V^{\prime 2}}}{\partial x}+\bar{v} \frac{\partial \overline{V^{\prime 2}}}{\partial r}+\overline{\boldsymbol{u}^{\prime}} \frac{\partial \overline{V^{\prime 2}}}{\partial x}+\overline{v^{\prime} \frac{\partial V^{\prime 2}}{\partial r}}+\overline{w^{\prime} \frac{\partial V^{\prime 2}}{r \partial \theta}}\right) \\
& \left.+\mu \overline{\left(\frac{\partial u^{\prime}}{\partial x}\right)^{2}}+\overline{\left(\frac{\partial v^{\prime}}{\partial x}\right)^{2}}+\overline{\left(\frac{\partial w^{\prime}}{\partial x}\right)^{2}}+\overline{\left(\frac{\partial u^{\prime}}{\partial r}\right)^{2}}+\overline{\left(\frac{\partial v^{\prime}}{\partial r}\right)^{2}}+\overline{\left(\frac{\partial w^{\prime}}{\partial r}\right)^{2}}+\overline{\left(\frac{\partial u^{\prime}}{r \partial \theta}\right)^{2}}+\overline{\left(\frac{\partial v^{\prime}}{r \partial \theta}\right)^{2}}+\overline{\left(\frac{\partial w^{\prime}}{r \partial \theta}\right)^{2}}\right\rceil
\end{aligned}
$$

Careful interpretation of the various terms in these equations is of paramount importance. The last three have been so arranged that the left-hand sides represent the rate of doing work and the right-hand sides the corresponding rate of energy change. The terms indicating the work done by pressure and by gravitational attraction are obvious, as are the rates of change of mean and turbulent kinetic energy $\rho \bar{V}^{2} / 2$ and $\rho \overline{V^{\prime 2}} / 2$ through convection by the mean motion 
and diffusion by the turbulence. Just as in the case of the purely viscous flow already discussed in brief detail, the work done by the stresses in turbulent flow is in part conservative and in part dissipative. Proceeding by analogy with the foregoing analysis of viscous flow, the conservalive terms stemming from the Reynolds equations have been replaced by the difference between total and dissipative terms and segregrated as in the first equation for the mean flow-total at the left and dissipative at the right. But the stresses now consist of both viscous and turbulent components, as rewritten in the alternative mean-flow equation. The significance of the dissipative part involving viscosity is the same as before. However, the part involving what are actually accelerative terms due to turbulent fluctuation cannot be truly dissipative, since it does not depend upon viscosityyet it is definitely non-conservative so far as the mean motion is concerned. In other words, the quantity

$$
-p\left[\overline{u^{\prime 2}} \frac{\partial \bar{u}}{\partial x}+\overline{v^{\prime 2}} \frac{\partial \bar{v}}{\partial r}+\overline{w^{\prime 2}} \frac{\bar{v}}{r}+\overline{u^{\prime} \bar{u}^{\prime}}\left(\frac{\partial \bar{u}}{\partial r}+\frac{\partial \bar{v}}{\partial x}\right)\right]
$$

represents the rate at which energy is irrecoverably lost by the mean motion and gained by the turbulent.

These turbulence-production terms appear again in the energy equation for the turbulent motion, but on the left-hand side, for they now represent an external supply of energy somewhat akin to work done by external forces. Work is again done by viscous stresses, but now those of the secondary motion. Finally, at the extreme right appear the terms representing the viscous dissipation within the eddies-by far the major part of the dissipation process.

The energy equation, like the several components of the momentum equation, becomes practically useful as it is integrated over the region under investigation. For the moment this operation will be indicated schematically in the form

$$
\begin{aligned}
& -\rho \int_{\boldsymbol{r}}\left(\bar{u} \frac{\partial \Omega}{\partial x}+\bar{v} \frac{\partial \Omega}{\partial r}\right) d \psi+\int_{\bar{F}} \frac{1}{r}\left[\frac{\partial}{\partial x} r\left(\overline{u \sigma}_{x}+{\overline{v \tau_{x}}}+\overline{w \tau}_{x \theta}\right)\right. \\
& \left.+\frac{\partial}{\partial r} r\left(\overline{u \tau}_{r x}+\overline{v \sigma}_{r}+\bar{w}_{r}\right)+\frac{\partial}{r \partial \theta} r\left(\overline{u \tau}_{\theta x}+{\overline{v \tau_{\theta r}}}+\overline{w \sigma}_{\theta}\right)\right] d \Psi \\
& =\frac{\rho}{2} \int_{\nabla}\left(\overline{u \frac{\partial V^{2}}{\partial x}}+\overline{v \frac{\partial V^{2}}{\partial r}}+\overline{w \frac{\partial V^{2}}{r \partial \theta}}\right) d \nvdash+\int_{\nabla}\left[\overline{\sigma_{x} \frac{\partial u}{\partial x}}+\overline{\sigma_{r} \frac{\partial v}{\partial r}}+\overline{\sigma_{\theta} \frac{\partial w}{r \partial \theta}}\right. \\
& \left.\overline{+\tau_{r \theta}\left(\frac{\partial v}{r \partial \theta}+\frac{\partial w}{\partial r}-\frac{w}{r}\right)}+\overline{\tau_{\theta x}\left(\frac{\partial w}{\partial x}+\frac{\partial u}{r \partial \theta}\right)}+\overline{\tau_{x r}\left(\frac{\partial u}{\partial r}+\frac{\partial v}{\partial x}\right)}\right] d H^{r}
\end{aligned}
$$

which, through application of the Gaussian transformation between volume and surface inlegrals, is written more significantly as

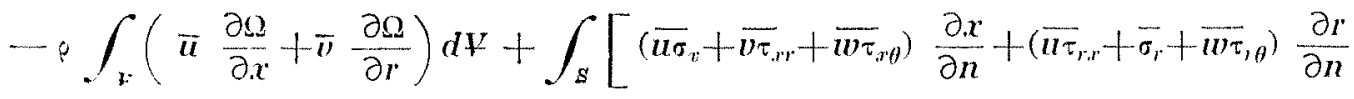

$$
\begin{aligned}
& \left.+\left(\bar{u} \tau_{i r}+\overline{v \tau_{\theta r}}+\overline{w \sigma_{\theta}}\right) \frac{r \partial \theta}{\partial n}\right\rceil d S=\frac{\rho}{2} \int_{S}\left(\bar{u} \overline{V^{2}} \frac{\partial x}{\partial n}+\overline{v V^{2}} \frac{\partial r}{\partial n}+\overline{w V^{2}} \frac{r \partial \theta}{\partial n}\right) d S
\end{aligned}
$$

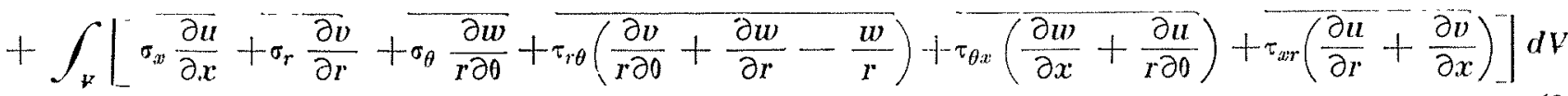

Herein the successive terms have much the same meaning as before-work on the left and energy transformation on the right. In evaluating the terms, it must be recalled that each velocity component consists of a mean and a fluctuating part, and each stress of a viscous and an inertial part, and that all products which are not necessarily zero have a place in the resulling expression.

\section{Interpretation in the Light of Isotropic Analysis}

The foregoing relationships have been seen to indicate a state of equilibrium among the processes of production, transport, and dissipation of lurbulent energy : production at the expense of the mean flow, transport by both convection and diffusion, and dissipation through the action of viscosity. In each of these processes the mean and fluctuating components of the velocity vector obviously play a primary role, but the only clue 
as to the scale at which the action occurs is found implicitly in the space derivatives of the components and their products. Quantitative evaluation of this scale is possible only through a statistical analysis of the velocity components as functions of space or time.

Each component of the velocity at any point of a turbulent flow will generally vary with time in a continuous though otherwise umpredictable manner, as illustrated in Fig. 7. If $f(u)$ represents the frequency with which the magnitude of the component $u$ lies between the values $u$ and $u+d u$, such that

$$
\int_{n}^{\infty} f(u) d u=1
$$

then the frequency distribution of the fluctuation is found to have roughly the form of the normal error curve shown in Fig. 8 . The mean value of the component corresponds to the abscissa of the centroid of the frequency area

$$
\bar{u}=\int_{0}^{\infty} u f(u) d u
$$

and the standard or root-mean-square deviation from this mean is represented by the radius of gyration of the frequency area about its centroidal axis :

$$
\sqrt{\overline{u^{2}}}=\left[\int_{0}^{\infty}(u-\bar{u})^{2} f(u) d u\right]^{1 / 2}
$$

Thus, whereas traces $A$ and $B$ of Fig. 7 have the same mean value, they are seen to differ considerably in their root-mean-square departure from the mean.

Trace $C$ evidently has the same mean as $A$ and $B$ and the same root-mean-square as $A$, but it differs from both in a manner that must be characterized by reference to the abscissa rather than the ordinate scale, through determination of the mean correlation $R_{t}$ between values the variable distance $\Delta t$ apart on the record; that is,

$$
R_{i}=\frac{\overline{u^{\prime}(t) u^{\prime}(t+\Delta t)}}{\overline{u^{\prime 2}}}
$$

As $\Delta t$ approaches zero, $R$, necessarily approaches unity (Fig. 9), but $R_{t}$ in turn approaches zero as $\Delta t$ exceeds a certain magnitude. If the deviation is small compared with the mean, the incremental time of the correlation function can be converted with close approximation to the corresponding incremental distance

$$
\Delta x \approx \bar{u} \Delta t
$$

whereupon the diagram of the correlation $R_{a}$ as illustrated in Fig. 10 becomes indicative of the scale of the secondary motion. Whether the point at which the correlation approaches zero or-preferably - the area under the diagram is used as a measure of this length, it is seen at once that traces $A$ and $B$ are characterized by a scale that is considerably larger than that of. $C$.

If one visualize the velocity fluctuations in fluid turbulence as the result of a heteregeneous system of eddies carried along by the mean flow, then the length scale obtained from the correlation function can be considered representative of the mean eddy size. That the eddy concept is not unreasonable is seen from the form of the correlation function for velocity components at right angles to the line connecting the points under consideration, illustrated by curve $g$ in Fig. 10; the function becomes negative for point spacings somewhat less than those at which curve $f$ approaches zero because, in effect, fluid on one side of an eddy tends to move upstream as that on the other side moves down.

Like the velocity components, the correlation function must be expected to vary with direction, for it depends upon the orientation of each of the two velocity components and upon that of the line between them-i.e., the separation vector $s$. Such double correlation is, in fact, a tensor function with nine different components, all of which are related to the scale of the eddy structure. (The Reynolds stresses, it should be noted, are limiting values of certain of these components for the condition of zero separation, and as such have lost their length significance.) Each component is necessarily a mean quantity which in itself gives no indication of the size distribution of the eddies actually involved in its determination.

Information of the latter sort is obtained by breaking down a velocity record like that of Fig. 7 into a spectral function showing the distribution of energy attributable to the given velocity component over the range of fluctuation frequencies or wave lengths encountered in the record. Such a function is, in fact, a Fourier transform of the correlation function [26]. However, the general spectrum has the same tensor nature as the general correlation, and it is the tensor rather than simply one of its components that is of present interest. If the mean kinetic energy of the turbulence per unit volume is recognized as the density times half the sum of the mean-square fluctuations, then this can be written as the integral of the energy per unit wave number $E(k)$ over the entire wave-number range :

$$
\frac{\rho}{2} \cdot\left(\overline{u^{\prime 2}}+\overline{v^{\prime 2}}+\overline{w^{\prime 2}}\right)=\int_{0}^{\infty} E(k) \mathrm{d} k
$$

Herein $k$ is the magnitude of the wave-number 
vector $[27]$-i.e. a tensor parameter equal to $2 \pi$ divided by a sort of spatial mean wave length. The essential point is that $k$ can thus be looked upon as the reciprocal of a length that characterizes the eddy scale. The resulting plot of such a spectrum (Fig. 11) therefore represents the distribution of energy among the various eddy sizes, these ranging from very large near the origin to very small at the far right.

For the special case introduced by Taylor [28] in which the turbulence is not only homogeneous (independent of position) but also isotropic (independent of direction), conditions are simplified to the extent that the meansquare fluctuations are all identical,

$$
\overline{u^{\prime 2}}=\overline{v^{\prime 2}}=\overline{w^{\prime 2}}
$$

their mean-square derivatives are half as great in the directions of the components as in the normal directions,

$$
\begin{aligned}
\overline{\left(\frac{\partial u^{\prime}}{\partial x}\right)^{2}} & =\overline{\left(\frac{\partial v^{\prime}}{\partial r}\right)^{2}}=\overline{\left(\frac{\partial w^{\prime}}{r \partial \theta}\right)^{2}} \\
& =\frac{1}{2} \overline{\left(\frac{\partial u^{\prime}}{\partial r^{\prime}}\right)^{2}}=\frac{1}{2} \overline{\left(\frac{\partial v^{\prime}}{r \partial \theta}\right)^{2}} \text { etc. }
\end{aligned}
$$

and the double-correlation tensor reduces to two interdependent functions [29]:

$$
g=f+\frac{s}{2} \frac{\partial f}{\partial s}
$$

The rate of dissipation of turbulent energy is hence expressible in terms of a single velocity derivative,

$$
\varepsilon=15 \mu \overline{\left(\frac{\partial u^{\prime}}{\partial x}\right)^{2}}
$$

and also in terms of the radius of curvature $\lambda$ of the correlation function $f$ of Fig. 10 at $R=1$ :

$$
\varepsilon=15 \mu \overline{\frac{u^{2}}{\lambda^{2}}}
$$

The general spectral function, moreover, can be reduced to yield the expression [25]

$$
\frac{\partial E(k)}{\partial t}=T(k)-2 \mu k^{2} E(k)
$$

which quite logically states that the rate at which the energy of a given wave number changes is equal to the net rate at which energy is gained through transfer from smaller to larger wave numbers minus the rate at which it is dissipated at that wave number.

Now the transfer of energy from one wave number to another is an inertial effect, whereas that of dissipation is viscous, and the ratio of the two should bence vary with the Reynolds number of the flow itself and with the relative position in the spectrum of the wave number under consideration. It was nevertheless hypothesized by Kolmogorofl [30] that at high Reynolds numbers the viscous effects would be concentrated in the very small eddies, and that the turbulence structure in this part of the spectrum would attain a condition of "universal" similarity or statistical equilibrium dependent upon only the kinematic rate of energy supply $S$ and the kinematic viscosity $v$, under which circumslances $S=\varepsilon / \rho$. This hypothesis has received considerable experimental vindication. It is of particular interest to note that the portion of the spectrum containing the major part of the energy (that shown in Fig. 11) is found to be quite distinct from that in which the dissipation occurs (which is actually well to the right of the part shown in the figure). 'That this situation is only to be expected can be seen from simple comparison of the quantities $E(k)$ and $k^{2} E(k)$ which characterize the two spectral zones.

Such conclusions with regard to homogeneous isotropic turbulence would be of little significance for the inhomogeneous, anisotropic phenomena under investigation were it not for two pertinent factors : first, there is sufficienl qualilative similarity between the two sorts of turbulence in general to serve a useful interpretive purpose; second, this similarity appears to assume quantitative aspects as the wave number increases. In any state of flow, for example, it is reasonable to conclude [25] that at all except low Reynolds numbers the structure of the large eddies depends almost wholly upon the geometry of the mean flow itself. Moreover, the higher the Reynolds number, the smaller should be the scale of the eddy motion at which viscosity begins to play in appreciable part. The stresses at scales greater than this are hence inertial, and their effect is to produce a continuous breakdown of the eddy motion in the direction of higher wave numbers. Therefore, just as the main flow is the source of eddies in regions of high velocity gradient, neighboring eddies may be visualized schematically as providing momentary zones of stress which engender still smaller eddies. The greater the range of scale over which this energy transfer has proceeded, the less evident the directional preferences of the original souree should have become. In other words, the finer the scale of particular eddies in a given turbulent field, the more nearly homogeneous and isotropic the motion within such eddies should be. At the same time, the dissipative effect of viscosity should increase as the scale decreases (in fact, approximately as the square of the wave number), and at sufficiently high Reynolds 
numbers practically all of the dissipation should be concentrated in that portion of the spectrum in which the turbulence is essentially isotropic.

The significant conclusion thus reached is that even in zones of inhomogeneous, anisotropic turbulence it may be possible to evaluate the rate of energy dissipation in terms of a single velocity derivative instead of the nine involved in the general dissipation function. There is already some evidence [31] that this conclusion is valid for relatively simple states of free-turbulence shear flow, though such is not necessarily the case for shear flow near boundaries $[32,33]$. Unfortunately, even considerably more refined studies than the following one cannot readily be used to provide substantiation of the theory for the more complex conditions which they involve, since practical limitations render unmeasurable still other terms which can only be presumed negligible. The assumption that the theory is valid is thus essential to the conduct of the analysis in its present state of approximation.

(To be continued)

\section{CORPORACION AUTONOMA REGIONAL DEL CAUCA CALI (COLOMBIE)}

\section{NOTE CONCERNANT LA QUALIFICATION PRÉALABLE DES ENTREPRENEURS}

Il est prévu que durant la seconde moitié de 1960, la Corporacion invitera un groupe sélectionné d'entrepreneurs préalablement qualifiés à soumissionner pour l'exécution du projet hydroélectrique de Calima, près de Cali.

Le projet comprendra un barrage en enrochements d'une hauteur maximum d'environ $100 \mathrm{~m}$ et un volume d'environ $2000000 \mathrm{~m}$; une centrale souterraine de $120000 \mathrm{~kW}$ avec un puits d'accès vertical de $230 \mathrm{~m}$ environ et un tunnel d'accès incliné d'environ $700 \mathrm{~m}$; un déversoir circulaire; une prise d'eau avec un puits pour vannes; une galerie d'amenée terminée par des conduites forcées enrobées dans des puits; un canal de fuite souterrain d'environ $1630 \mathrm{~m}$; un tunnel de dérivation de $660 \mathrm{~m}$ environ; un tunnel d'adduction de $7 \mathrm{~km}$; ainsi que des ouvrages accessoires.

Les frrmes intéressées qui ont déjà réalisé d'une manière satisfaisante des travaux comparables sont invitées à demander des formulaires à remplir pour leur qualification préalable auprès de la Corporacion Autonoma Regional Del Cauca. Apartado Aereo 2366, Cali, Colombie. Les formulaires de qualification remplis devront être retournés avant le 15 juillet 1960. Les soumissions ne seront acceptées que de la part de soumissionnaires préalablement qualifiés. 